Please cite this article as: M. La Cerva, M. Ciofalo, L. Gurreri, A. Tamburini, A. Cipollina, G. Micale, On some issues in the computational modelling of spacer-filled channels for membrane distillation. Desalination 411 (2017) 101-111. doi: 10.1016/j.desal.2017.02.016

\title{
ON SOME ISSUES IN THE COMPUTATIONAL MODELLING OF SPACER-FILLED CHANNELS FOR MEMBRANE DISTILLATION
}

\author{
Mariagiorgia La Cerva, Michele Ciofalo*, Luigi Gurreri, \\ Alessandro Tamburini, Andrea Cipollina, Giorgio Micale \\ Dipartimento dell'Innovazione Industriale e Digitale (DIID) \\ Università degli Studi di Palermo, Viale delle Scienze, 90128 Palermo, Italy
}

\begin{abstract}
This study addresses issues which arise in the computational and experimental modelling of flow and heat / mass transfer in membrane distillation and other processes adopting spacer-filled channels (either planar or spiral wound), but have not been sufficiently clarified in the literature so far. Most of the argumentations presented are based on original computational results obtained by the authors by finite volume simulations; some literature results are also considered where appropriate. The main questions addressed regard the choice of scales for the reduction of data and the definition of dimensionless numbers ( $\mathrm{Re}, f, \mathrm{Nu}, \mathrm{Sh}$ ); the definition of average heat or mass transfer coefficients; the combined effects of the main parameters that characterize the process (notably spacer pitch to channel height ratio $l / H$, flow attack angle $\gamma$ and Reynolds number Re) and the applicability of simple correlations; the influence of the spacer's thermal conductivity. In regard to the complex influence of the parameters, $\mathrm{Re}, l / H$ and $\gamma$ were found to interact heavily, making a separate-effect analysis impossible and power-law friction or heat / mass transfer correlations of little use. Thermal conduction in the spacer, even for low-conductivity polymeric spacers $\left(\lambda \approx 0.15 \mathrm{Wm}^{-1} \mathrm{~K}^{-1}\right)$, was found to be responsible for up to $10 \%$ of the total heat transfer;
\end{abstract}

Keywords: Membrane Distillation; Spacer Filled Channel; Computational Fluid Dynamics; Heat Transfer; Mass Transfer.

*Corresponding author; email: michele.ciofalo@unipa.it 


\section{INTRODUCTION}

\subsection{Membrane-based processes}

Compared to traditional fluid treatment technologies, membrane processes offer significant advantages, among which a compact and modular design, the ability selectively to transfer specific components, a high energy efficiency and a moderate waste production [1].

In the present paper, the attention is on Membrane Distillation (MD) and other membrane processes adopting flat (plane or spirally wound) channels, generally provided with spacers. In such systems, understanding the fluid dynamics in the channels is crucial both for enhancing mixing (in order to reduce undesirable polarization effects) and for limiting pressure drop (in order to reduce pumping power requirements and structural issues). Other arrangements (e.g. dead-end or tubular and hollow-fiber membranes) raise flow and mixing problems of a different kind and are beyond the scope of the present work.

In $\mathrm{MD}$, the transferred species is water vapor and the driving force is the temperature difference between feed and permeate, of the order of 5 to $50 \mathrm{~K}$. The feed channel thickness usually ranges from 2 to $5 \mathrm{~mm}$ and the cross flow velocity from 5 to $20 \mathrm{~cm} / \mathrm{s}$, yielding Reynolds numbers (based on the hydraulic diameter of the channels) ranging from 200 to 2000. MD has been the subject of several experimental and computational studies carried out by our group at the University of Palermo in the context of international research programs [2-6].

\subsection{Polarization phenomena}

Membrane processes are inherently affected by polarization phenomena which cause a decrease of the driving forces and thus a loss of efficiency. Usually, separation membrane processes exhibit concentration polarization. In Membrane Distillation, the effects of concentration polarization are usually negligible compared to that of temperature polarization: this phenomenon results in a temperature difference at the membrane interfaces smaller than the temperature difference between the bulk solutions (Figure 1). The loss of efficiency in the process is often quantified by introducing a temperature polarization coefficient (TPC), defined as:

$$
T P C=\frac{T_{f m}-T_{p m}}{T_{f b}-T_{p b}}
$$

in which " $f$ " and " $p$ " refer to feed and permeate while " $m$ " and " $b$ " refer to membrane surface and fluid bulk, respectively. This coefficient increases with the Reynolds number, especially for low Re, and decreases with the heat flux [7]. 


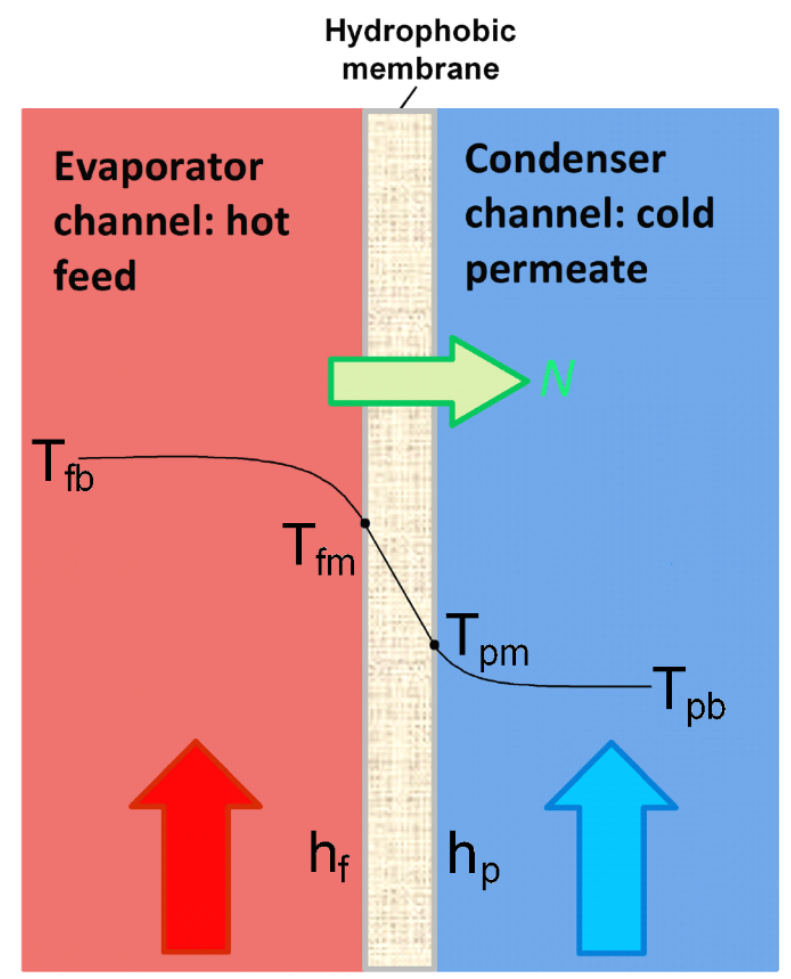

Figure 1 Sketch of the temperature polarization effect in Membrane Distillation.

The negative effects of polarization phenomena can be counteracted using mixing promoters, which usually serve also as spacers keeping a fixed distance between opposite membranes. There are many studies in the literature on the characterization of spacer-filled channels, mostly focused on the investigation of the effect of spacer geometry (filament diameter, filament arrangement, filament spacing, angle between crossing filaments, flow attack angle) on fluid dynamics features such as recirculation, flow regime, flow pattern, shear rate distribution, mass and heat transport phenomena, and pressure drops.

Figure 2(a) shows a general overlapped spacer geometry, formed by stacking two arrays of cylindrical rods, or filaments: the most general case is characterized by diameters $d_{1}, d_{2}$, pitches $l_{1}, l_{2}$, included angle $\alpha$ and flow attack angle $\gamma$. Neglecting contact deformation or partial compenetration of the filaments, the channel height $H$ can be assumed equal to $d_{1}+d_{2}$. Figure 2(b) shows a woven spacer geometry; a unit cell is reported for the particular case $d_{1}=d_{2}, l_{1}=l_{2}=6 d, \alpha=90^{\circ}$. Other types of spacers have also been investigated. 


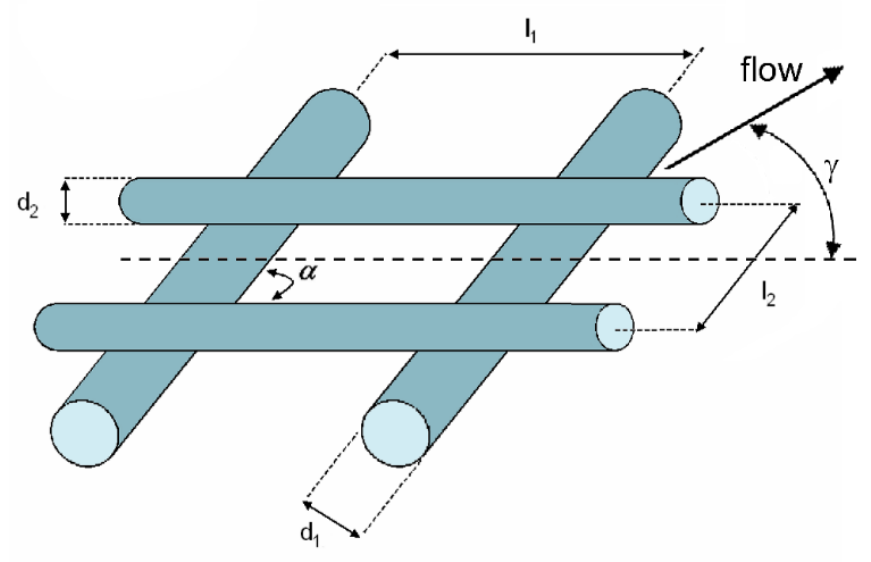

(a)

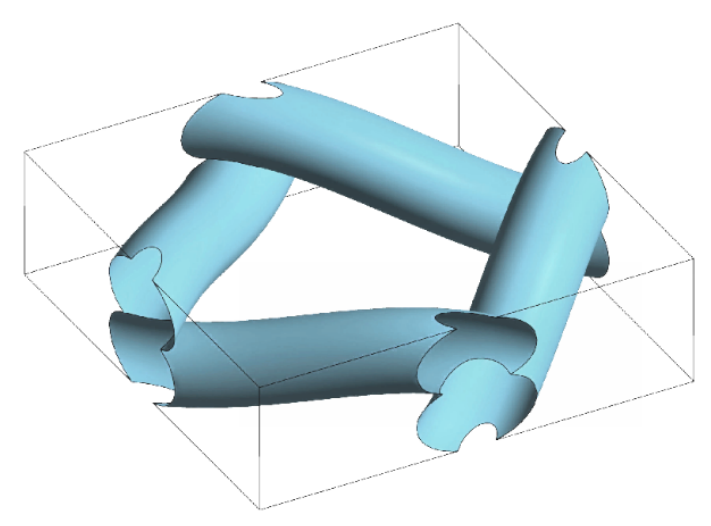

(b)

Figure 2 (a) General overlapped spacer geometry, formed by stacking two arrays of cylindrical rods, or filaments: the most general case is characterized by diameters $d_{1}, d_{2}$, pitches $l_{1}, l_{2}$, included angle $\alpha$ and flow attack angle $\gamma$. (b) Woven geometry; a unit cell is shown for the case $d_{1}=d_{2}, l_{1}=l_{2}=6 d, \alpha=90^{\circ}$.

\section{THE APPLICATION OF CFD TO SPACER-FILLED CHANNELS}

\subsection{Review of the literature}

While a thorough discussion of the choice of dimensionless numbers and of the definition of average heat / mass transfer coefficients is postponed to sections 3 and 4, in the following, in order to make comparisons easier, the Reynolds number Re, the Darcy friction coefficient $f$ and the Nusselt or Sherwood numbers $\mathrm{Nu}$, Sh will be based on twice the channel height $(2 H)$ as the length scale and on the inlet, or approach, velocity $u_{v o i d}$ as the velocity scale, independent of the (possibly different) choices made by the authors of the various papers examined.

Applications of Computational Fluid Dynamics to the characterization of spacer-filled channels for membrane processes started to appear in the literature with some consistency in the 1990s. Many of the published studies regarded simplified, two-dimensional geometries, e.g. cylindrical obstacles placed in a plane channel [8-11]. Although they may provide valuable insight into the basic mechanisms of flow separation and drag, 2-D studies can only deal with transverse filaments and are not adequate to predict the complex mixing and heat / mass transfer enhancement caused by real 3-D spacers.

In 2001 Karode and Kumar [12] presented purely hydrodynamic CFD simulations for a multicell model of the ultrafiltration spacer-filled channels investigated experimentally by Da Costa et al. [13]. The authors considered overlapped spacers in which the pitch (inter-filament distance) to channel height ratio ranged between 1 and 4 and the Reynolds number between $\sim 500$ and $\sim 5000$. Steady-state flow was assumed in all cases. The PHOENICS ${ }^{\mathrm{TM}}$ CFD code was used. The main factors influencing the effectiveness of a spacer were found to be the pitch to channel height ratio and the 
angle between the spacer filaments. For the highest inlet flow rates, simulations overestimated the pressure drop compared to that measured experimentally; Karode and Kumar attributed this discrepancy to the non-ideality of the actual spacers that could allow some fluid flow between the filaments and the membrane.

Li et al. [14] used the CFX-4.3 $3^{\mathrm{TM}}$ code to study, for a non-woven geometry, the influence on mass transfer of pitch (distance between parallel filaments) $l$, angle $\alpha$ between filaments and flow attack angle $\gamma$. They carried out direct numerical simulations (DNS) for Reynolds numbers of $\sim 200$ 1000, a range in which spiral wound membrane modules may actually operate. The authors defined the average mass transfer coefficient as the area average of the local mass transfer coefficient. The results, in terms of Sherwood number in function of Power number $(P n)$, showed the spacer geometry to be optimal for $l / H=4, \alpha=120^{\circ}$ and $\gamma=30^{\circ}$.

Koutsou et al. [15] performed DNS at $\operatorname{Re}<800$ using Fluent ${ }^{\mathrm{TM}}$. The computational domain was a periodic unit cell. The authors highlighted that a transition to unsteady flow occurs at Reynolds numbers of 140-180 for the parameter range examined. They also found the presence of closed recirculation zones attached to the spacer filaments, presumably detrimental for concentration polarization and fouling. In a later paper, Koutsou et al. [16] investigated Reynolds and Schmidt number effects on the time-mean local and spatially-averaged Sherwood number, taking into consideration various realistic spacer geometries. They carried out 3D DNS with the same Reynolds number range as in [15] and Schmidt numbers in the range 1-100. A local time-mean value of the mass transfer coefficient $(k)$ at the channel walls was defined on the basis of the local time-mean value of the wall mass flux and of the local time-mean concentration at the channel symmetry plane. In order to calculate the overall mean Sherwood number, the authors used the spatially averaged value of $k$. They found that the distributions of the local time-mean Sherwood number at the top and bottom walls were symmetrical with respect to the channel diagonal, which was also the direction of the mean flow.

Koutsou et al. [17] developed a novel spacer geometry consisting of spheres of diameter equal to the channel height $H$ connected by cylindrical segments of diameter $H / 2$. They considered the periodic unit cell formed by four neighbouring spherical nodes and connecting segments and performed DNS using Fluent ${ }^{\mathrm{TM}}$. The main advantage of this novel geometry is that the contact of the spherical spacer nodes with the membrane surfaces occurs pointwise, unlike in conventional geometries characterized by contact lines that create "dead" zones associated with reduced mass transfer rates. The authors observed the same general trends of results obtained with conventional spacers, but, regarding the local shear stresses and mass transfer coefficients on the membrane 
surface, they found that the novel geometry exhibits higher values and a more uniform spatial distribution of these parameters.

Cipollina et al. [2] studied spiral wound MD channels with double layer filament spacers using either DNS or steady state simulations. The computational domain included 5-6 unit cells both streamwise and spanwise and was meshed by a tetrahedral unstructured finite volume grid. The code used was Ansys-CFX ${ }^{\mathrm{TM}}$. The authors imposed $\mathrm{Re} \approx 400$, developing flow and thermal fields along the unit cells starting from uniform inlet velocity and temperature (i.e., inlet-outlet conditions with no periodicity), and a uniform heat flux on both walls. They concluded that higher velocities reduce temperature polarisation while filaments transverse to the flow increase polarisation. In a further paper [3], the authors extended the study to a larger variety of non-woven spacers. The results confirmed that filaments mainly parallel to the fluid flow direction are to be preferred, and showed that 3- and 4-layer spacers provide a better compromise between low $\Delta p$ and low temperature polarization than diamond or oblique 2-layer spacers.

Al-Sharif et al. [18] studied the effect of three different overlapped spacers on fluid dynamic, heat transfer and temperature polarization using OpenFOAM ${ }^{\circledR}$. Like Cipollina et al. [2, 3], they adopted a developing flow approach, i.e. they considered a certain number of unit cells in the axial direction and applied uniform profiles for velocity and temperature at the inlet of the domain and zero gradient conditions at the outlet. A uniform heat flux boundary condition was imposed on both walls. From the results of simulations for $\mathrm{Re} \approx 300-1200$, the authors stated that spacers characterized by 3 layers of orthogonal cylindrical filaments placed at $0^{\circ}$ (filaments touching the walls) and $90^{\circ}$ (central filaments) with respect to the flow (type 3) caused the lowest pressure drop, symmetrical temperature profile and high velocities near the membrane walls. Spacers with two layers of orthogonal cylindrical filaments placed at $0^{\circ}-90^{\circ}$ with respect to the flow (type 2) were the least desirable as they produced asymmetric temperature and velocity profiles and high pressure drops. An intermediate behavior was reported for spacers with a flow attack angle of $45^{\circ}$ (type 1 ).

Also Mojab et al. [19] used OpenFOAM ${ }^{\circledR}$ to investigate by DNS laminar to turbulent flow regimes in a channel provided with an overlapped spacer, which consisted of two layers of mutually orthogonal rods, or filaments, placed at $45^{\circ}$ with respect to the flow direction. The filament spacing was just 1.085 times the channel height, equal in its turn to twice the rod diameter. This configuration was identical to the commercial spacer CONWED-1 $1^{\mathrm{TM}}$. The authors used the periodic unit cell approach with no-slip conditions at the spacer and membrane surfaces. They found that the main flow splits into two streams which move parallel to the filaments with $90^{\circ}$ direction difference to each other. At the cell midplane these streams interact and exchange momentum. The $90^{\circ}$ angle between the flow streams produces tangential forces which cause secondary swirling motions in the main flow 
streams. The authors found four different flow regimes: laminar-steady $(\operatorname{Re}<480)$, laminar-unsteadyperiodic $(\operatorname{Re}=480-720)$, fully unsteady $(\operatorname{Re}>720)$ and turbulent flow proper $(\operatorname{Re}>2400)$.

Saeed et al. [20] used Fluent ${ }^{\mathrm{TM}}$ to investigate the impact of dimensionless filament mesh spacing on wall shear stress and mass transfer coefficients for the two membrane walls. They employed steady state and laminar flow conditions and considered as the computational domain a strip of unit cells including six bottom filaments and one top filament. The average mass transfer coefficient was defined as the area average of the local mass transfer coefficient. The authors compared different spacers with pitch to channel height ratios $(l / H)$ varying between 2 and 6; cases with different values of $l / H$ for the top and bottom layer of filaments were also considered. The Reynolds number (based, in this case, on hydraulic diameter and mean interstitial velocity) ranged between 75 and 200 (if computed on the basis of the present definition, Re would vary in a complex way as a function of the pitch to channel height ratios of each filament layer). In all simulations the flow direction was parallel to the top filaments. The authors observed closed flow recirculation near the bottom membrane surface for spacer geometries characterized by $l / H \leq 3$. For spacers with $l / H \geq 4$ they observed reattachment and separation regions, with a reattachment point location that shifted downstream with an increase in Re, until it met the next bottom filament. Finally, they found that the spacer arrangement with $l / H=4$ on both walls provides moderate pressure drops and the highest Spacer Configuration Efficacy (defined as the ratio of the Sherwood number to the power number).

With the aim of characterizing water flow in a spacer filled channel, Bucs et al. [21] performed Particle Image Velocimetry (PIV) in a transparent test section provided with a commercial non-woven Toray ${ }^{\mathrm{TM}}$ spacer with a thickness of $0.787 \mathrm{~mm}, l / H=5.56$, a flow attack angle of $45^{\circ}$ and filaments of diameter varying along their axis. They also simulated the unit cell of the experimental geometry, reconstructed from computerized tomography (CT) scans. The authors assumed steady laminar flow and $\operatorname{Re} \approx 112,256$ and 480. The measured flow was laminar with only a slight unsteadiness in the upper velocity limit, and CFD simulations were in good agreement with the measured flow fields.

Tamburini et al. [4] studied the effects of spacer orientation on heat transfer in MD channels provided with non-woven spacers using DNS, the unit cell approach and the Ansys-CFX ${ }^{\mathrm{TM}}$ code. In order to reproduce their own experimental measurements as faithfully as possible, the authors considered only one of the walls (top wall) to be thermally active (i.e., representing a membrane) and imposed on it a third-type (mixed) boundary condition with an outer temperature of $19^{\circ} \mathrm{C}$ and an interposed thermal resistance $r_{\text {ext }}$ of $6.25 \cdot 10^{-3} \mathrm{~m}^{2} \mathrm{~K} / \mathrm{W}$. The opposite (bottom) wall and the filament surfaces were assumed to be adiabatic. The average Nusselt number $\langle\mathrm{Nu}\rangle$ was defined as the area average of the local $\mathrm{Nu}$ on the active wall. The authors found that, when the fluid moves at a $45^{\circ}$ angle with respect to the filaments adjacent to the top wall, $\langle\mathrm{Nu}\rangle$ is higher than in the $0^{\circ}$ and $90^{\circ}$ 
orientations. They also compared CFD predictions with their own experimental results, obtained by Liquid Crystal Thermography, and found a good agreement in the time averages of both local and surface-averaged Nusselt numbers.

Gurreri et al. [22] used Ansys-CFX ${ }^{\mathrm{TM}}$ to simulate woven (w) and overlapped (o) spacers with three pitch to height ratios $(l / H=2,3,4)$, two different flow directions (either parallel to a filament, $\alpha=0$, or bisecting the angle formed by the filaments, $\left.\alpha=45^{\circ}\right)$ and four Reynolds numbers $(1,4,16$, 64). Such low Re are representative of flow in Reverse Electrodialysis (RED) stacks. They found that the filament spacing has a clear effect on Sh for all Re only in woven spacers with $\alpha=45^{\circ}$ (in the range investigated, Sh decreases as $l / H$ increases), while for the overlapped arrangement the dependence of Sh on $l / H$ is not as significant. The authors stated that the woven arrangement establishes different flow fields than those typical of overlapped spacers, raising pressure drop but favoring mixing, especially for higher Reynolds numbers and $\alpha=45^{\circ}$. Finally, they found that the woven spacer with $\alpha=45^{\circ}$ is the most promising configuration for mixing; it yields higher values of Sh than the case $\alpha=0^{\circ}$ for all Re and $l / H$, although the improvements are lower at high Re.

\subsection{Settled and unsettled issues in the numerical modelling of spacer-filled channels}

As the above, fairly complete, survey shows, CFD simulations of spacer-filled channels for membrane processes started to appear in the literature since the year 2001 and have been presented at an increasing rate since then. They have been based, in most cases, on a small number of open source or commercial codes (OpenFOAM, Ansys-CFX ${ }^{\mathrm{TM}}$, Ansys-Fluent ${ }^{\mathrm{TM}}$ and, in one case, PHOENICS ${ }^{\mathrm{TM}}$ ), since in-house codes are poorly suitable for the complex geometry of spacer-filled channels. Published studies are almost evenly divided between those using the Periodic Unit Cell approach [4, 14-17, 19, 21-22] and those simulating multi-cell, developing flow configurations [2, 3 , 9, 11, 12, 18, 20]. Further distinctions can be drawn according to the 2-D [8, 9, 11] versus 3-D [2-4, 12,14-22] dimensionality of the simulations and to the flow regimes investigated: thus, some authors carried out only steady-state, laminar simulations [3, 9, 11, 19, 21-22]; others dealt with unsteady and early turbulent regimes by Direct Numerical Simulation [2, 4, 14-16, 19]; and others yet [8, 11] used turbulence models (RNG $k-\varepsilon$ in the former case, Spalart-Allmaras and $k-\omega$ in the latter). In regard to the phenomena modelled, some papers $[12,15,19,21]$ simulated only the fluid flow, while others considered also heat transfer [2-4, 9, 11, 18] or mass transfer [16-17, 20, 22].

Schwinge et al. [23], Ghidossi et al. [24], Hitsov et al. [25], Fimbres-Wehis and Wiley [26] and Karabelas et al. [27] have presented reviews addressing a number of issues in the modeling of spacerfilled channels. In particular, Fimbres-Weihs and Wiley [26] examined problems concerning the 
definition of the dimensionless numbers that characterize the phenomenon, the use of periodic conditions, and the comparison of 3D vs. 2D and steady-state $v s$. transient simulations.

The present paper aims to complement the above reviews, by discussing a number of aspects not sufficiently clarified so far. The main issues addressed here are:

- $\quad$ choice of scales to compute dimensionless numbers;

- definition of average heat / mass transfer coefficients;

- $\quad$ influence of the thermal conductivity of the spacer;

- $\quad$ complex influence of the parameters $l / H, \gamma, \mathrm{Re}$.

Most of the results that follow make reference to heat transfer in Membrane Distillation (MD) and were obtained for the spacer geometry Diamond_90 (Figure 3), consisting of identical overlapping cylindrical filaments (Figure 2(a)) with $d_{f}=H / 2$ and an intrinsic angle $\alpha=90^{\circ}$, under steady-state (laminar) flow assumptions. All simulations were conducted by the finite volume code Ansys CFX. Numerical methods and computational details have been discussed elsewhere [4, 22].

\section{CHOICE OF SCALES TO COMPUTE DIMENSIONLESS NUMBERS}

\subsection{Scales for the Reynolds number}

In regard to the Reynolds number, several definitions can be found in the literature, differing in the choice of the length and velocity scales.

The length scale has been variously identified with the filament diameter $d_{f}$, the channel height $H$, or the hydraulic diameter $d_{h}=4 V_{f} / S_{\text {wet }}$, in which $V_{f}$ is the volume of fluid and $S_{w e t}$ the wetted surface (including both the filaments and the membrane walls). A further alternative consists in using the hydraulic diameter of the void channel, which can be identified with twice its height $(2 H)$ in the limit of laterally infinite plane channel.

As to the velocity scale, some authors prefer the volume-averaged velocity $u_{m}$ along the main flow direction $s$, which coincides with the area average of $u_{s}$ on any arbitrary cross section orthogonal to the flow and is sometimes called the interstitial velocity:

$$
u_{m}=\frac{1}{V_{f}} \int_{V_{f}} u_{s} \mathrm{~d} V
$$

An alternative velocity scale is the void channel velocity $u_{\text {void }}$, i.e. the velocity which would yield the given flow rate if the channel were void of any spacer; $u_{\text {void }}$ is sometimes called the inlet, approach or superficial velocity. The two quantities $u_{m}$ and $u_{\text {void }}$ are related by

$$
u_{\text {void }}=u_{m} \mathcal{E}
$$

where $\varepsilon$ is the porosity, or void ratio, $V_{f} / V_{t o t}$. 
Thus, at least the following quantities (covering seven out of a total of eight possible combinations!) have been called the "Reynolds number" in the literature:

- $\quad \operatorname{Re}_{(1)}=u_{m} d_{h} / v[2,3,11-12,19-21]$

- $\quad \operatorname{Re}_{(2)}=u_{\text {voidd }} / v[18]$

- $\quad \operatorname{Re}_{(3)}=u_{v o i d} H / v[18]$

- $\quad \operatorname{Re}_{(4)}=u_{\text {void }} 2 H / v[4,6,22]$

- $\quad \operatorname{Re}_{(5)}=u_{m} H / v[14]$

- $\quad \operatorname{Re}_{(6)}=u_{\text {void }} d_{f} / v[15-17]$

- $\quad \operatorname{Re}_{(7)}=u_{m} 2 H / v[5]$

In particular, in a spacer with overlapped filaments of diameter $d_{f}$, distance between the filaments $l$ and channel height $H=2 d_{f}$, one has

$$
\begin{gathered}
\varepsilon=\frac{l / H-\pi / 8}{l / H} \\
d_{h}=2 H \varepsilon \frac{l / H}{l / H+\pi / 2}
\end{gathered}
$$

(independent of the angle formed by the filaments with each other and with the main flow), so that between the above definitions of the Reynolds number the following relations apply:

$$
\operatorname{Re}_{(1)}=\frac{1}{\varepsilon} \operatorname{Re}_{(2)}=2 F \operatorname{Re}_{(3)}=F \operatorname{Re}_{(4)}=2 \varepsilon F \operatorname{Re}_{(5)}=4 F \mathrm{Re}_{(6)}=\varepsilon F \mathrm{Re}_{(7)}
$$

in which $F=(l / H) /(l / H+\pi / 2)$. Similar formulae can be written for spacers characterized by woven filaments or by more complex configurations.

Definition $\operatorname{Re}_{(1)}$ is by far the most popular. Definitions $\operatorname{Re}_{(3)}$ and $\operatorname{Re}_{(4)}$, differing only by a factor 2, are based on velocity and length scales that do not depend on the spacer and are proportional to the flow rate through the channel, which allows a more direct performance comparison between different spacers (including the "null" case of a void, i.e. spacerless, channel). Note that, for $l / H \rightarrow \infty$, one has $F \rightarrow 1$ and $\operatorname{Re}_{(4)} \rightarrow \operatorname{Re}_{(1)}$.

\subsection{Scales for the friction coefficient}

With some exceptions [2-3, 8-9, 19,21], most of the literature on spacer-filled channels deals to some extent with measured or predicted pressure drops. Some authors $[11,18]$ choose to report this quantity in dimensioned form (i.e., in $\mathrm{Pa}$ or $\mathrm{Pa} / \mathrm{m}$ ), or use the dimensioned or dimensionless wall shear stress [15-16] or the power number, related to the pumping power consumption per unit volume [14]. 
However, the commonest choice $[4,12,20,22]$ is to make the pressure drop $\Delta p$ dimensionless as a Darcy friction coefficient $f$ on the basis of the usual relation

$$
f=\frac{\Delta p}{\Delta s} \frac{2 L_{r e f}}{\rho U_{r e f}^{2}}
$$

in which $\Delta s$ is the distance travelled along the main flow direction $s$, while $L_{r e f}$ and $U_{r e f}$ are the reference length and velocity, respectively. Note that $f$ is four times the Fanning coefficient. The reported values of $f$ depend sensitively on the choice of $L_{r e f}$ and $U_{r e f}$. Normally, they are the same used to define the Reynolds number, but this is not always true; for example, Saeed et al. [20] evaluate Re from $u_{m}$ and $d_{h}$ but compute $f$ from $u_{m}$ and $2 H$.

The definitions adopted have an even larger influence on $f$ than they have on $\mathrm{Re}$, due to the $U$ square term in Eq.(7). For example, adopting $L_{r e f}=d_{h}, U_{r e f}=u_{m}$ to compute $f_{(1)}$ and $L_{r e f}=2 H, U_{r e f}=u_{\text {void }}$ to compute $f_{(4)}$, from the above formulae for overlapped spacers it follows that $f_{(1)} / f_{(4)}=0.291$ for $l / H=2$, 0.527 for $l / H=4$, and 0.8 (still far from 1) for $l / H=12$. One also has $\operatorname{Re}_{(1)} / \operatorname{Re}_{(4)}=0.560$ for $l / H=2,0.718$ for $l / H=4$, and 0.884 for $l / H=12$. Since $f_{(1)}<f_{(4)}$ and simultaneously $\operatorname{Re}_{(1)}<\operatorname{Re}_{(4)}$, while $f$ is normally a decreasing function of $\mathrm{Re}$, plotting $f_{(1)}$ against $\operatorname{Re}_{(1)}$ yields a curve far below the corresponding plot of $f_{(4)}$ against $\operatorname{Re}_{(4)}$. These differences should be kept in mind when comparing results from different literature sources.

\subsection{Scales for the Nusselt or Sherwood number}

With a few exceptions $[2-3,18]$, most of the studies dealing with heat / mass transfer in spacer-filled channels express the relevant results in dimensionless form by introducing a Nusselt or a Sherwood number. While the exact definition of the local or averaged heat / mass transfer coefficients themselves will be discussed in section 4 below, the influence of the scales chosen for their conversion into dimensionless Nusselt / Sherwood numbers is also worth a brief discussion.

Considering, for example, heat transfer, one has

$$
\mathrm{Nu}=\frac{h L_{r e f}}{\lambda}
$$

in which $h$ is the heat transfer coefficient (ratio of a heat flux to a temperature difference), $L_{r e f}$ is the reference length scale, and $\lambda$ is the fluid's thermal conductivity. For mass transfer, $\mathrm{Nu}$ is replaced by $\mathrm{Sh}, h$ is replaced by the mass transfer coefficient $k$ (ratio of a mass flux to a concentration difference), and $\lambda$ is replaced by the kinematic diffusivity $D$. If, coherently, one defines $\mathrm{Nu}$ on the basis of the same length scale used for the definition of Re and $f$, then different Nusselt numbers are obtained, which, following the above nomenclature for Re, may be classified as 
- $\quad \mathrm{Nu}_{(1)}=\mathrm{Nu}_{(2)}=h d_{h} / \lambda[9,11,20,26]$

- $\quad \mathrm{Nu}_{(3)}=\mathrm{Nu}_{(5)}=h H / \lambda[14]$

- $\quad \mathrm{Nu}_{(4)}=h 2 H / \lambda[4,22]$

- $\quad \mathrm{Nu}_{(6)}=h d_{f} / \lambda[16-17]$

(with obvious changes when mass transfer is considered).

Also in this case, the different definitions should be carefully considered when comparing results from different literature sources.

\section{DEFINITION OF LOCAL OR AVERAGED HEAT / MASS TRANSFER COEFFICIENTS}

\subsection{Local coefficients}

Making reference to heat transfer, and assuming the heat flux to be positive if it exits the channels (cooled fluid), the most common definition of the local heat transfer coefficient is:

$$
h=\frac{q_{w}^{\prime \prime}}{T_{b}-T_{w}}
$$

In Eq. (9) $q^{\prime \prime}$ is the local heat flux, $T_{w}$ is the local wall temperature and $T_{b}$ is the bulk temperature, usually defined as:

$$
T_{b}=\frac{\int_{A} \rho c_{p} T u_{s} \mathrm{~d} A}{\int_{A} \rho c_{p} u_{s} \mathrm{~d} A}
$$

$A$ being the area of a generic cross section of the channel and $u_{s}$ the velocity component along the main flow direction $s$. Often $\rho$ and $c_{p}$ can be treated as constant properties. Note that $T_{b}$ remains a function of $s$. In some circumstances, notably involving complex three-dimensional geometries, the association of the wall temperature at a given point on the surface with the bulk temperature at a specific abscissa $s$ is rather arbitrary. Moreover, in many simulations adopting the unit cell approach, only the periodic component of the bulk temperature, not varying from cell to cell, is simulated. In these cases it may be preferable to define the bulk temperature by replacing the area integrals in Eq. (10) with volume integrals taken over the whole unit cell. Yet another alternative is to replace the bulk temperature in the definition (9) of $h$ with a different reference value, e.g. the fluid's temperature at the channel midplane [16] or the fluid's inlet temperature [11].

In the case of mass transfer, temperature is replaced by concentration and $h$ is replaced by a mass transfer coefficient $k$. 
The problem with the above definition of $h$ or $k$ is that, in complex geometries involving flow recirculation, these coefficients may easily become singular or negative in some regions of the wall. This occurs whenever the iso-surface $T=T_{b}$ (or $C=C_{b}$ ) touches the wall; $h(k)$ diverges on the line $T_{b}=T_{w}\left(C_{b}=C_{w}\right)$ and becomes negative inside this line. An example is reported in Figure 3, showing the iso-surface $T=T_{b}$ (bulk temperature) for an overlapped spacer with $l / H=4, \gamma=0^{\circ}, \operatorname{Re} \approx 42$ and twoside heat transfer with uniform imposed wall heat flux. Even in the absence of singularities, the simple fact that the iso-surface $T=T_{b}$ (or $C=C_{b}$ ) approaches the wall closely may give rise to abnormal and unphysically large values of $h$ or $k$. This kind of distribution is rather common, especially for imposed flux (Neumann) or mixed (third-type) boundary conditions, two-side heat / mass transfer, high Reynolds numbers and high Prandtl / Schmidt numbers. The only situation in which singularities cannot occur are those characterized by imposed wall temperature or concentration (Dirichlet boundary conditions).

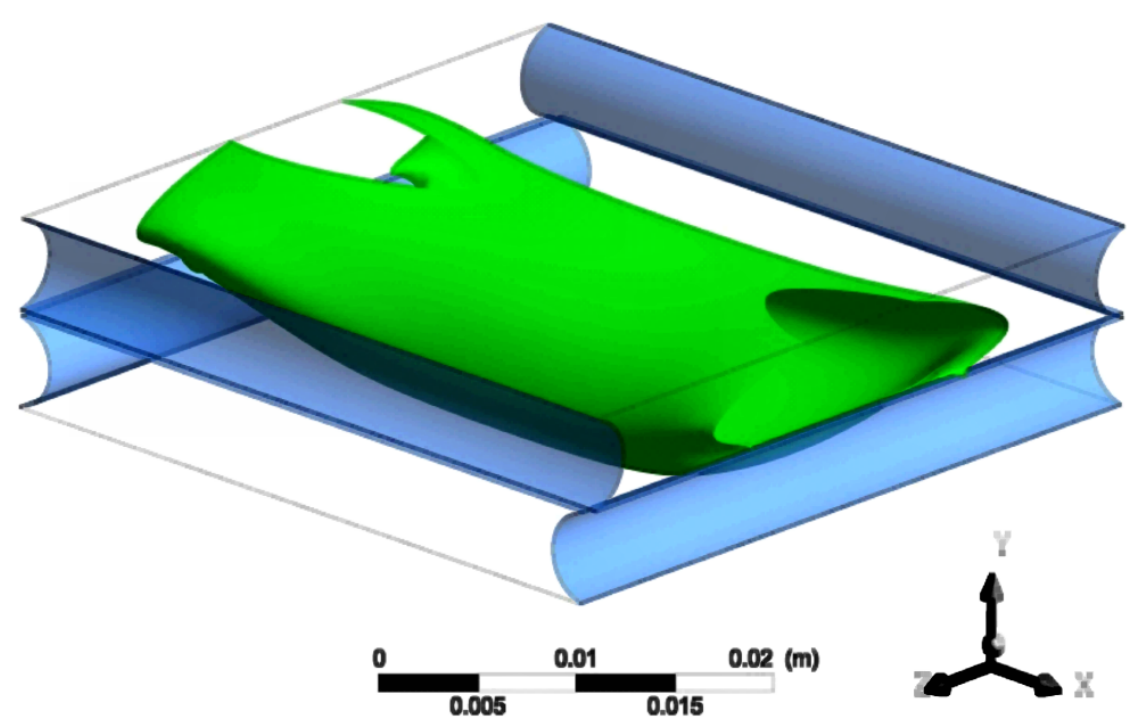

Figure 3 Iso-surface $T=T_{b}$ (bulk temperature) for an overlapped spacer with $l / H=4, \gamma=0^{\circ}, \operatorname{Re} \approx 42$ and two-side heat transfer with uniform imposed wall heat flux.

Several computational studies dealing with heat or mass transfer prefer to avoid the complications related to the definition of $h$ or $k$ and mention only temperatures or concentrations and heat / mass fluxes.

\subsection{Average coefficients}

In most papers dealing with heat or mass transfer in spacer-filled channels $[9,14,16-17,20]$, the average coefficient is simply defined as the area average 


$$
h^{(1)}=\langle h\rangle=\frac{1}{S} \int_{S} h \mathrm{~d} S=\frac{1}{S} \int_{S} \frac{q_{w}^{\prime \prime}}{T_{b}-T_{w}} \mathrm{~d} S
$$

where $S$ may be the whole active transfer surface or a sub-region of it (e.g., one of two active channel walls). Obvious modifications apply if mass transfer is considered.

The problem with this definition is that, if the local $h$ (or $k$ ) is singular or attains unphysically very high or negative values (for the reasons discussed in the previous section), also its average $\langle h\rangle$ (or $\langle k\rangle$ ) will be affected by this behavior and will attain anomalous values. A definition of the averages that does not suffer from this problem is $[4,22]$

$$
h^{(2)}=\frac{\left\langle q_{w}^{\prime \prime}\right\rangle}{T_{b}-\left\langle T_{w}\right\rangle}
$$

in which $\left\langle q{ }^{\prime \prime}\right\rangle$ and $\left\langle T_{w}\right\rangle$ are surface averages of $q{ }_{w}$ or $T_{w}$ over the surface of interest (e.g., one of the walls or both walls). The usual obvious modifications apply in the case of mass transfer. Corresponding dimensionless numbers $\mathrm{Nu}^{(1)}, \mathrm{Nu}^{(2)}$ or $\mathrm{Sh}^{(1)}, \mathrm{Sh}^{(2)}$ can be obtained from the above definitions by using Eq. (8) or its mass transfer equivalent, provided a length scale has been chosen.

$\mathrm{Nu}^{(1)}$ and $\mathrm{Nu}^{(2)}$ coincide when a uniform wall temperature is imposed, while they are different when a uniform wall heat flux or a wall thermal resistance are imposed. In fact, one can write:

$$
\mathrm{Nu}^{(1)}=\text { constant } \times\left\langle\frac{q_{w}^{\prime \prime}}{\Delta T_{b w}}\right\rangle, \quad \mathrm{Nu}^{(2)}=\text { constant } \times \frac{\left\langle q_{w}^{\prime \prime}\right\rangle}{\left\langle\Delta T_{b w}\right\rangle}
$$

in which $\Delta T_{b w}=T_{b}-T_{w}$. When $T_{w}$ is imposed, $\Delta T_{b w}$ is a constant (say, $\Delta T_{b w}{ }^{0}$ ) and one has

$$
\mathrm{Nu}^{(1)}=\mathrm{Nu}^{(2)}=\operatorname{constant} \times \frac{\left\langle q_{w}^{\prime \prime}\right\rangle}{\Delta T_{b w}^{0}}
$$

On the contrary, when a uniform heat flux is imposed, $q{ }_{w}$ is a constant (say, $\left.q{ }^{\prime \prime}{ }^{0}\right)$ and one has

$$
\mathrm{Nu}^{(1)}=\text { constant } \times q_{w}{ }^{0} 0\left\langle\frac{1}{\Delta T_{b w}}\right\rangle \neq \mathrm{Nu}^{(2)}=\mathrm{constant} \times q_{w}{ }^{0} \frac{1}{\left\langle\Delta T_{b w}\right\rangle}
$$

since the average of the reciprocal is not the reciprocal of the average. 


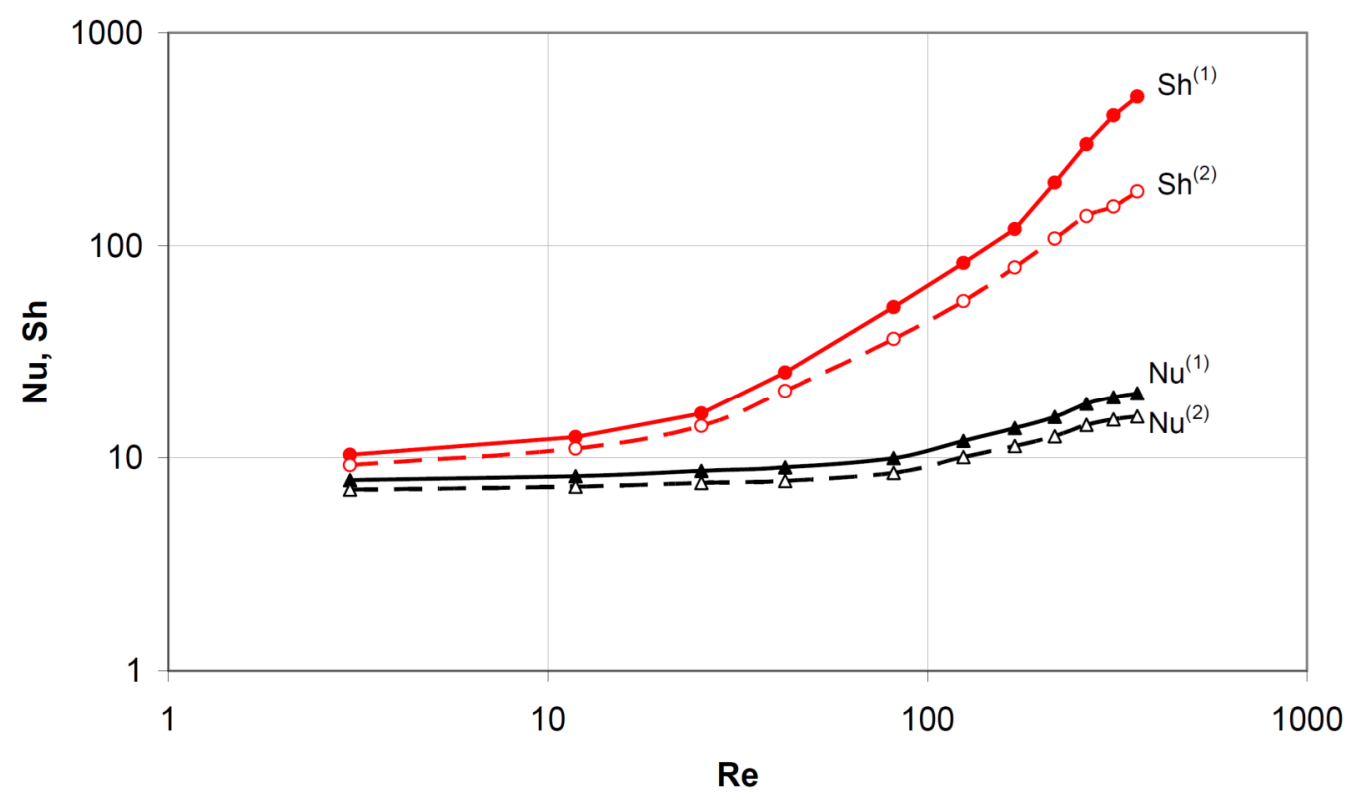

Figure 4 Average Nusselt and Sherwood numbers, computed by definitions (1) and (2), as functions of the Reynolds number for an overlapped spacer with $l / H=4$, flow attack angle $\gamma=45^{\circ}$, two-side heat $/$ mass transfer and third-type boundary conditions (Prandtl number $\operatorname{Pr}=4$, Schmidt number Sc=600).

The difference between the two definitions is illustrated in Figure 4, which reports average Nusselt and Sherwood numbers, computed by definitions (1) and (2), as functions of the Reynolds number for an overlapped spacer with $l / H=4$, a flow attack angle $\gamma=45^{\circ}$ and two-side heat $/$ mass transfer. The values 4 and 600 were assumed for the Prandtl and Schmidt numbers, respectively. Third type boundary conditions were imposed at the walls. It can be observed that $\mathrm{Nu}^{(2)}$ and $\mathrm{Sh}^{(2)}$ are always lower than $\mathrm{Nu}^{(1)}$ and $\mathrm{Sh}^{(1)}$, and that the difference increases with Re. It can also be observed that the difference between $\mathrm{Sh}^{(1)}$ and $\mathrm{Sh}^{(2)}(\mathrm{Sc}=600)$ is larger than that between $\mathrm{Nu}^{(1)}$ and $\mathrm{Nu}^{(2)}(\mathrm{Pr}=4)$.

\section{COMPLEX INFLUENCE OF THE PARAMETERS $l / H, \gamma, \operatorname{Re}$}

In this section, some considerations will be presented concerning the individual influence of the main spacer parameters that affect heat / mass transfer in membrane processes. The objective is to clarify to what extent this influence can be represented by simple correlations suitable for design purposes, especially by separated-effect power-law correlations of the kind commonly used in engineering [10, 28]. In particular, the quantities $l / H$ (spacer pitch to channel height ratio), $\gamma$ (flow attack angle) and Re (Reynolds number) will be considered.

Simulations were carried out for overlapped non-conducting spacers with orthogonal filaments (intrinsic angle $\alpha=90^{\circ}$ ), two-side heat transfer, and third-type thermal boundary conditions at both walls. One parameter at a time among $l / H, \gamma$ and Re was made to vary. Simulations were limited to laminar, steady-state conditions so that no turbulence model was used. 


\subsection{Influence of the Reynolds number}

In regard to the influence of the Reynolds number, Figure 5(a) reports the average Nusselt number $\mathrm{Nu}^{(2)}$ at both walls as a function of Re for $l / H=4$ and three values of the flow attack angle $\gamma\left(0^{\circ}, 20^{\circ}\right.$ and $\left.45^{\circ}\right)$. Note that, for $\gamma=45^{\circ}, \mathrm{Nu}^{(2)}$ top $=\mathrm{Nu}^{(2)}$ bottom. On the whole, a monotonic increase of $\mathrm{Nu}^{(2)}$ with Re can be observed, with some reservations concerning the bottom-wall value for $\gamma=0^{\circ}$ at low Reynolds numbers; however, the rate of variation of $\mathrm{Nu}^{(2)}$ with $\mathrm{Re}$ is not uniform, but depends on the flow attack angle and on the wall considered; this suggests that separated-effect correlations would perform poorly. The situation is even worse for the smaller aspect ratio $l / H=2.5$, Figure $5(\mathrm{~b})$. Here, $\mathrm{Nu}^{(2)}$ is far from monotonic, but rather exhibits a plateau on either wall and at all attack angles; however, the level attained and the Reynolds number range in which $\mathrm{Nu}^{(2)}$ remains approximately constant depend on the specific wall and angle $\gamma$ considered, and a simple fit of the results appears prohibitive. Results of comparable complexity are obtained by considering other aspect ratios and flow attack angles. Note that a plateau or even a decrease in $\mathrm{Nu}$ when expressed as a function of $\mathrm{Re}$ is not surprising since, as the fluid's velocity varies, the length of the wake and recirculation regions created downstream of each obstacle also varies, so that the flow interaction with the subsequent obstacles and with the walls changes in a complex way, and may either promote or impair heat / mass transfer.

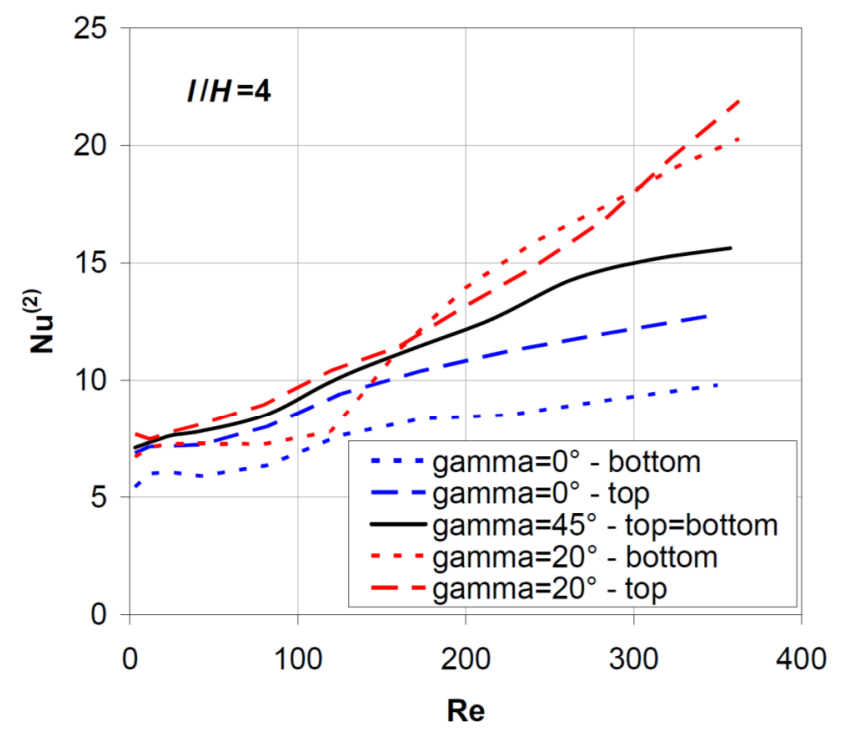

(a)

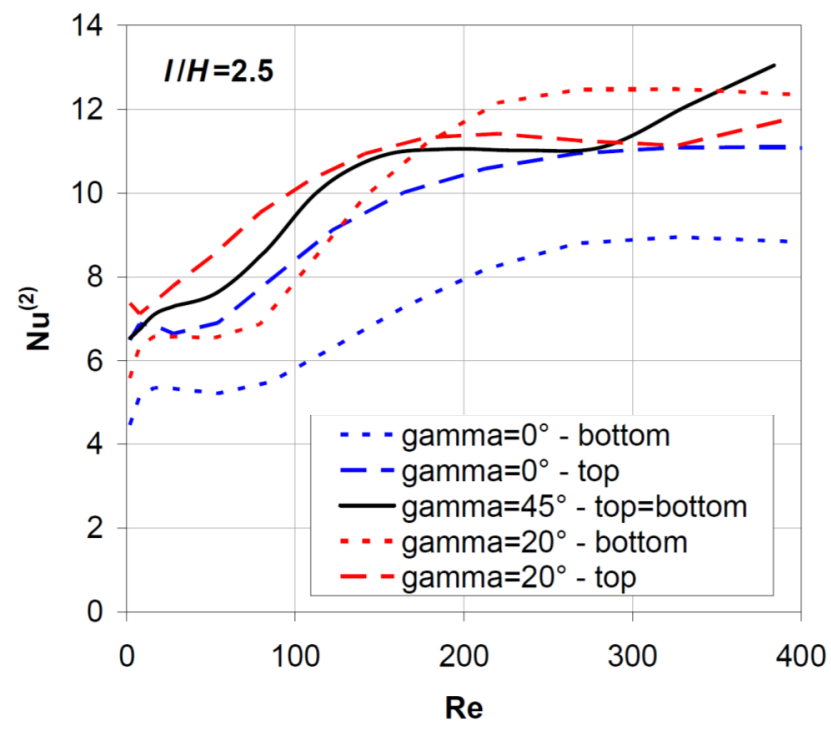

(b)

Figure 5 Influence of the Reynolds number on heat transfer: average Nusselt numbers $\mathrm{Nu}^{(2)}$ on top and bottom walls as functions of Re for overlapped spacers, two-side heat transfer and different flow attack angles $\gamma$. (a) $l / H=4$; (b) $l / H=2.5$. 


\subsection{Influence of the spacer pitch to channel height ratio}

In regard to the influence of the spacer pitch to channel height ratio $l / H$ (aspect ratio) on pressure drop, Figure 6 reports the friction coefficient $f$ (normalized by the friction coefficient in the corresponding spacerless channel, $f_{s}=96 / \mathrm{Re}$ ) as a function of $l / H$ in overlapped spacers for two different values of the flow attack angle $\gamma\left(0^{\circ}\right.$ and $\left.45^{\circ}\right)$ and two different values of the Reynolds number $\operatorname{Re}\left(20\right.$ and 300). Note that $f$ does not change if $\gamma$ varies from $0^{\circ}$ to $90^{\circ}$. The figure shows that $f$ decreases markedly with $l / H$ (of course, one expects $f / f_{s} \rightarrow 1$ for $l / H \rightarrow \infty$ ); it also shows that the influence of the flow attack angle $\gamma$ (better discussed in the following section) is negligible for the lower Reynolds number (20), while it is significant at $\mathrm{Re}=300$, when inertial effects become important.

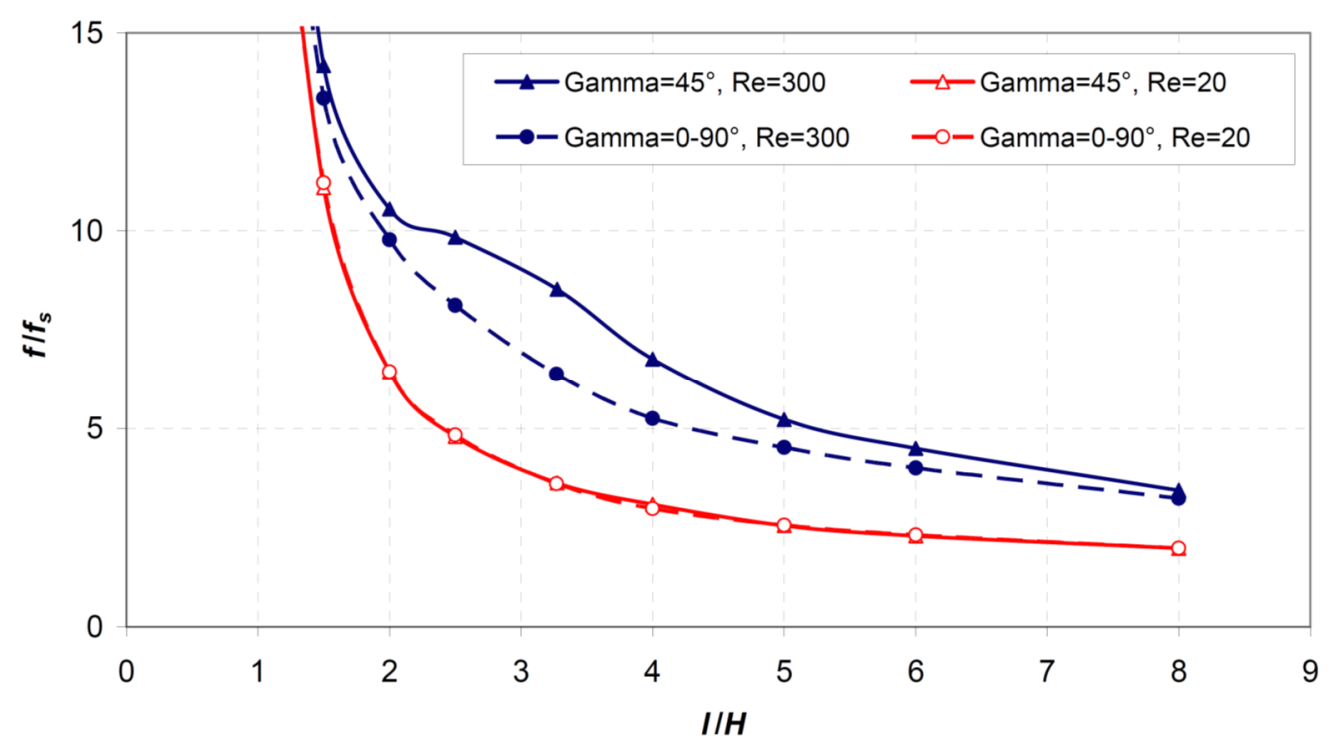

Figure 6 Influence of the spacer pitch to channel height ratio on pressure drop: friction coefficient $f$ (normalized by 96/Re) as a function of $l / H$ in overlapped spacers for two different values of the flow attack angle $\gamma\left(0^{\circ}\right.$ and $\left.45^{\circ}\right)$ and two different values of the Reynolds number $\operatorname{Re}(20$ and 300). Note that identical values of $f$ are obtained for $\gamma=0^{\circ}$ and $90^{\circ}$.

In order to illustrate the influence of $l / H$ on heat / mass transfer, the behavior of the average Nusselt number $\mathrm{Nu}^{(2)}$ as a function of $l / H$ for two values of $\operatorname{Re}(20$ and 300$)$ and $\gamma=45^{\circ}$ is reported in Figure 7 (a). The choice of this flow attack angle makes the values of $\mathrm{Nu}^{(2)}$ on the two walls identical, so that a single value needs to be reported. It can be observed that, for the larger Reynolds number of 300 , close to those expected in $\mathrm{MD}$ applications, $\mathrm{Nu}^{(2)}$ possesses a marked maximum at $l / H \approx 3.5$, in accordance with most of the literature. A second noteworthy feature of the results is that, for $l / H>4$, 
$\mathrm{Nu}^{(2)}$ decreases slowly with $l / H$, and still exhibits values only $\sim 25 \%$ lower than the maximum for $l / H=6$. Taking account of the strong reduction in the friction coefficient associated with an increase in $l / H$, see Figure 6 , high aspect ratio spacers are potentially attractive candidates in an optimization perspective.

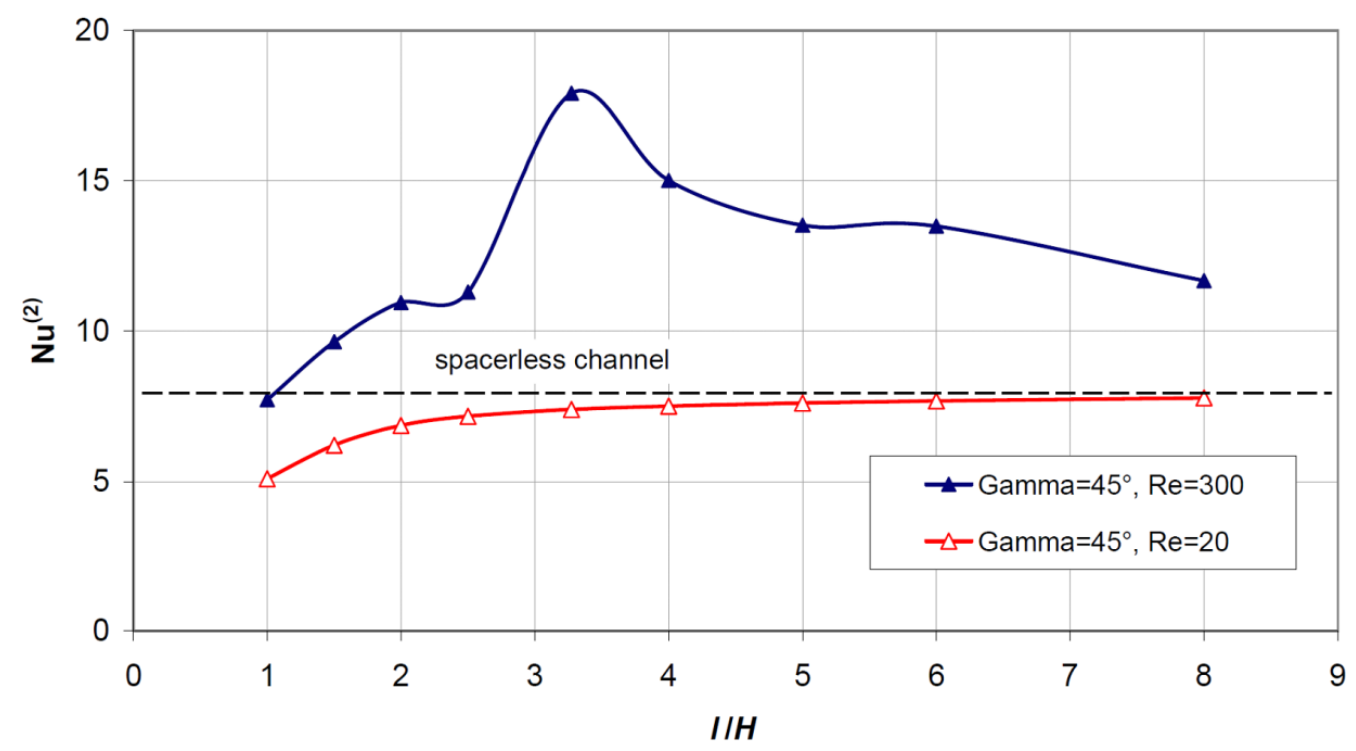

(a)

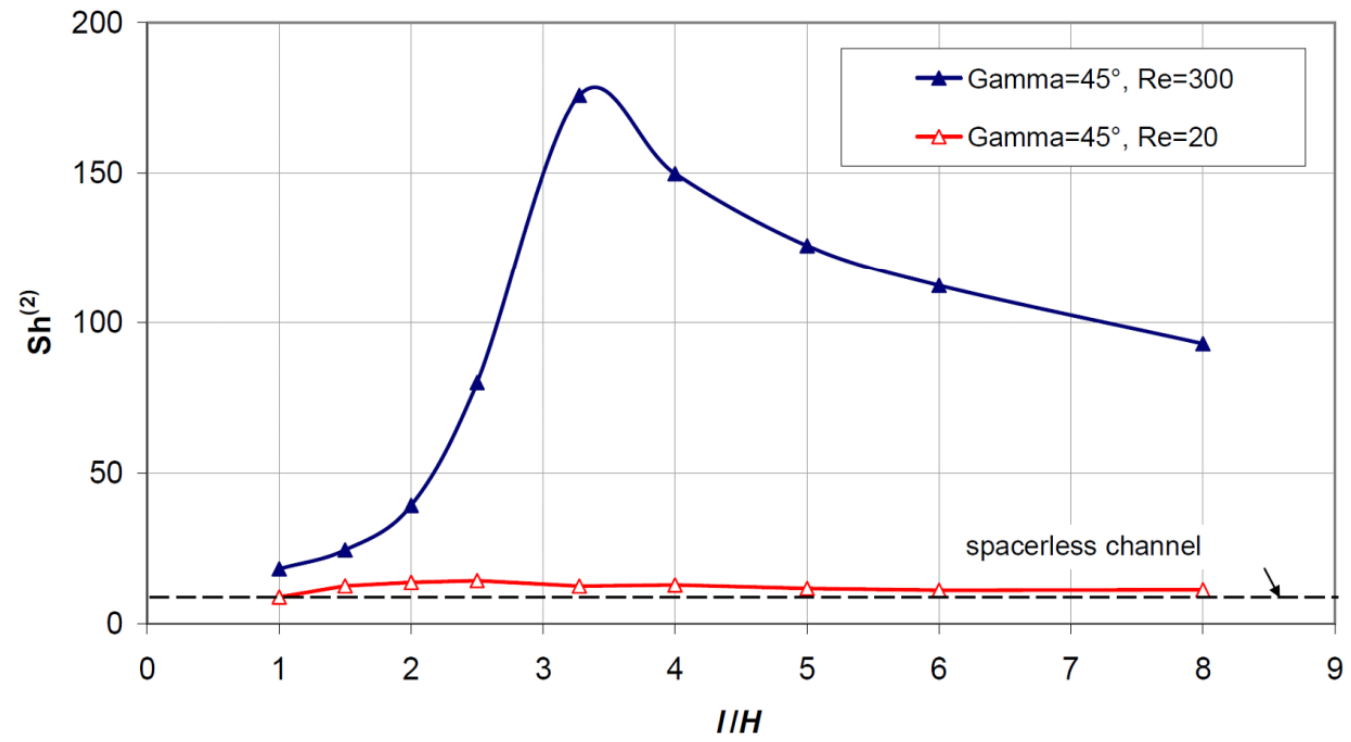

(b)

Figure 7 Influence of the spacer pitch to channel height ratio on heat transfer: average Nusselt number $\mathrm{Nu}^{(2)}$ (a) and Sherwood number $\mathrm{Sh}^{(2)}$ (b) as functions of $l / H$ for overlapped spacers, two-side heat transfer, $\gamma=45^{\circ}$ and two different values of $\operatorname{Re}(20$ and 300).

In regard to the viability of simple correlations, the complex dependence of $\mathrm{Nu}^{(2)}$ upon $l / H$ shown by Figure 7(a) for $\mathrm{Re}=300$ makes this possibility rather unlikely. At the lower Reynolds number, $\mathrm{Nu}^{(2)}$ 
is always lower than the value ( 8) obtained in a spacerless plane channel and increases with $\operatorname{Re}$ tending to this spacerless value for $l / H \rightarrow \infty$ (as it must be at any Re for physical consistency). This means that at very low Re, in the absence of significant secondary flow, the spacer acts mainly as an obstacle to heat transfer rather than as a promoter.

Figure 7(b) reports the corresponding behavior of the average Sherwood number $\mathrm{Sh}^{(2)}$, computed for a Schmidt number of 600 (chosen as representative of sodium chloride in Direct or Reverse Electrodialysis) and a bulk concentration of $\sim 0.5 \mathrm{M}$. For $\mathrm{Re}=300$, the behavior of $\mathrm{Sh}^{(2)}$ is similar to that of $\mathrm{Nu}^{(2)}$ and exhibits its maximum at the same $l / H$ ratio $(\sim 3.5)$. As a difference, for the lower Reynolds number $\mathrm{Sh}^{(2)}$ remains slightly higher than the spacerless plane channel value of $\sim 8$ (which, in laminar flow, is independent of the Schmidt number and thus is identical to $\mathrm{Nu}^{(2)}$ ), and exhibits an asymptotic approach from above to this value for $l / H \rightarrow \infty$.

\subsection{Influence of the flow attack angle}

In regard to the variation of the Nusselt number with the attack angle $\gamma$, most of the experimental and 3-D computational studies presented so far consider only a few orientations, with the main flow parallel or orthogonal to the filaments, or bisecting the angle formed by these latter $[3,4,12,15-19$, 21-22] (an orientation cannot even be defined in the case of 2-D numerical simulations [8, 11]). Yet, the few studies considering different attack angles [14], as well as physical intuition, suggest that orientations other than the basic ones mentioned above may offer advantages in terms of heat / mass transfer and frictional pressure drop, and thus are worth investigating.

Preliminarily it should be observed that, for each spacer configuration, symmetries exist such that the influence of $\gamma$ needs not to be studied in the whole interval $0-2 \pi$, but in a narrower range. Consider, for example, the overlapped geometry sketched in Figure 8, in which the two arrays of wires are assumed to be mutually orthogonal and to have the same diameters and pitches. The flow attack angle $\gamma$ can be conventionally defined as the angle formed by the main flow direction with the filaments touching the top wall (reference direction $x$ ). Alternative definitions have also been used in the literature.

A moment's reflection shows that, for symmetry considerations, the following properties hold for any quantity $\Phi$ that can be defined as an average over either the top or the bottom wall (e.g. pressure, shear stress, temperature, concentration, heat or mass flux, heat or mass transfer coefficient, Nusselt or Sherwood number):

$$
\Phi_{\text {wall }}(\gamma)=\Phi_{\text {wall }}(\gamma+k \pi) \quad(" \text { wall" = "top" or "bottom"; } k=0, \pm 1, \pm 2, \ldots)
$$




$$
\Phi_{\text {wall }}(\gamma)=\Phi_{\text {wall }}(\pi-\gamma) \quad(" \text { wall" = "top" or "bottom" })
$$

and, provided the same boundary conditions apply on the top and bottom wall,

$$
\Phi_{\text {top }}(\gamma)=\Phi_{\text {top }}\left(\frac{\pi}{2}-\gamma\right)
$$

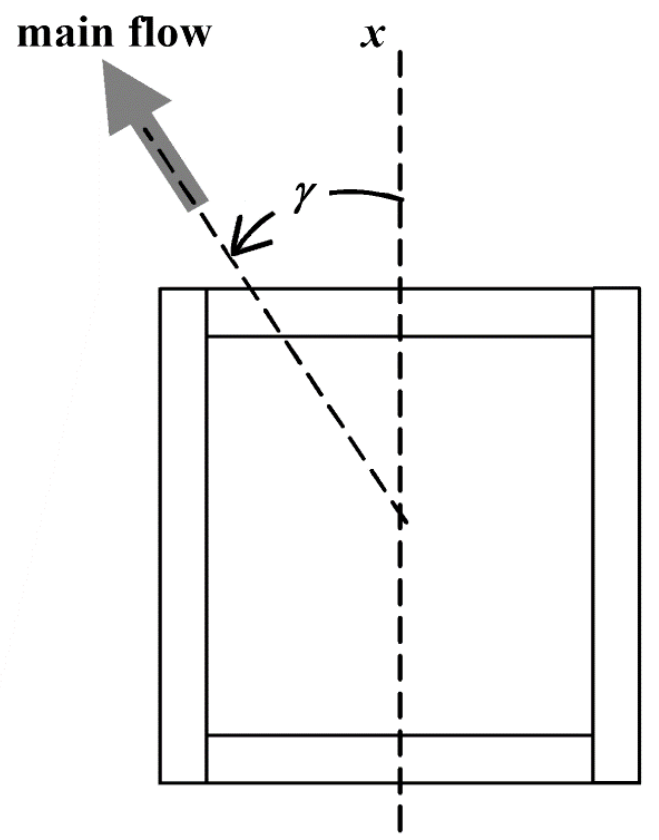

Figure 8 Sketch of the unit cell of an overlapped spacer-filled channel, showing the reference filament direction and the flow attack angle $\gamma$.

Thanks to the above properties, a knowledge of the behavior of both top- and bottom-wall average quantities in the interval $\gamma=0-\pi / 4$ (obtained either experimentally or computationally) is sufficient for the whole curves in $0-2 \pi$ and for both walls to be built. Two consequences of eqs. (16)-(18) are:

$$
\begin{aligned}
& \frac{\partial \Phi_{\text {wall }}}{\partial \gamma}=0 \quad \text { for } \gamma=k \frac{\pi}{2}(" \text { wall" }=\text { "top" or "bottom"; } k=0, \pm 1, \pm 2, \ldots) \\
& \Phi_{\text {top }}\left(\frac{\pi}{4}\right)=\Phi_{\text {bottom }}\left(\frac{\pi}{4}\right)
\end{aligned}
$$

If a quantity $\Psi$ is obtained by averaging over the whole (top+bottom) wall surface, then, as a consequence of the above symmetry properties, its dependence on $\gamma$ is periodic with period $\pi / 2$ and satisfies the following properties:

$$
\frac{\partial \Psi}{\partial \gamma}=0 \quad \text { for } \gamma=k \frac{\pi}{4}(k=0, \pm 1, \pm 2, \ldots)
$$




$$
\Psi(\gamma)=\Psi\left(\frac{\pi}{2}-\gamma\right)
$$

Examples are the overall average Nusselt number, $\mathrm{Nu}^{(2)}{ }_{a v g}=\left[\mathrm{Nu}^{(2)}{ }_{t o p}+\mathrm{Nu}^{(2)}{ }_{b o t t o m}\right] / 2$ and the corresponding average Sherwood number $\mathrm{Sh}^{(2)}$ avg. Properties (21)-(22) hold also for the friction coefficient $f$.

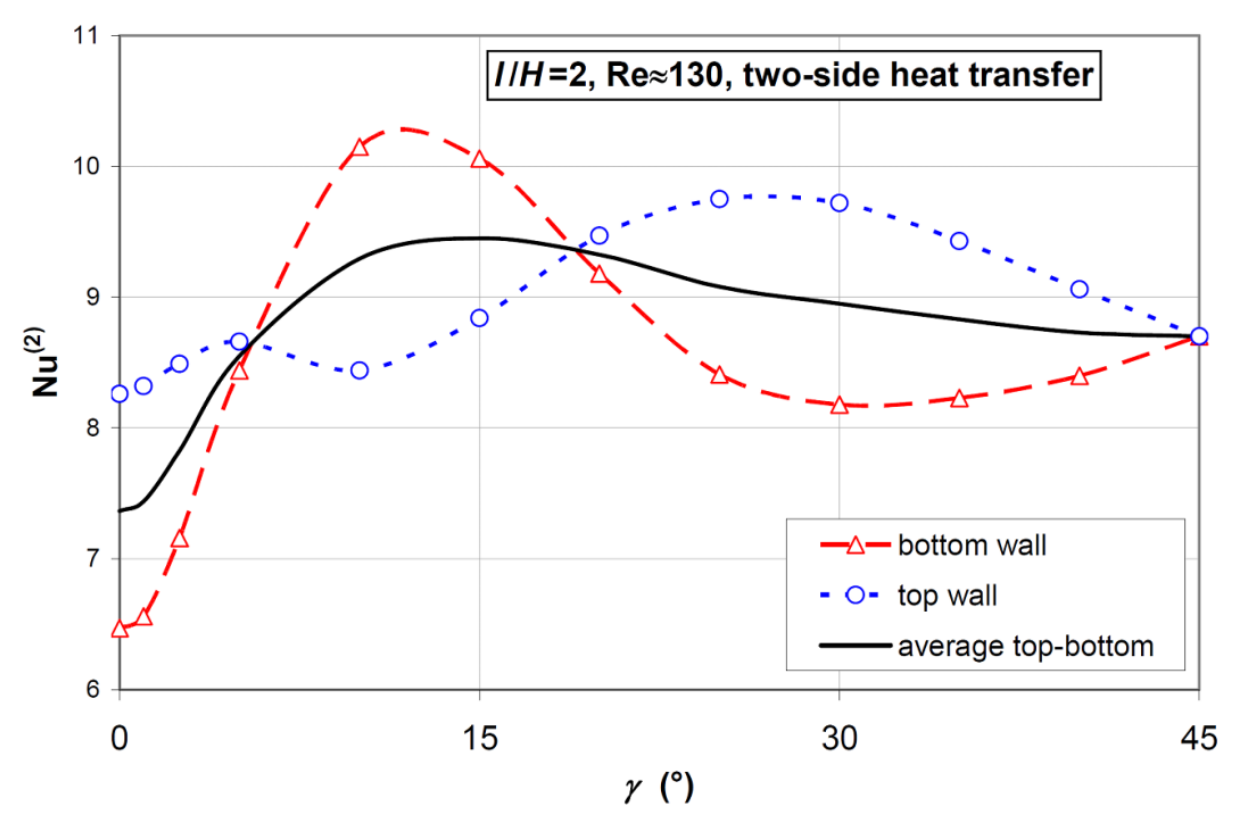

(a)

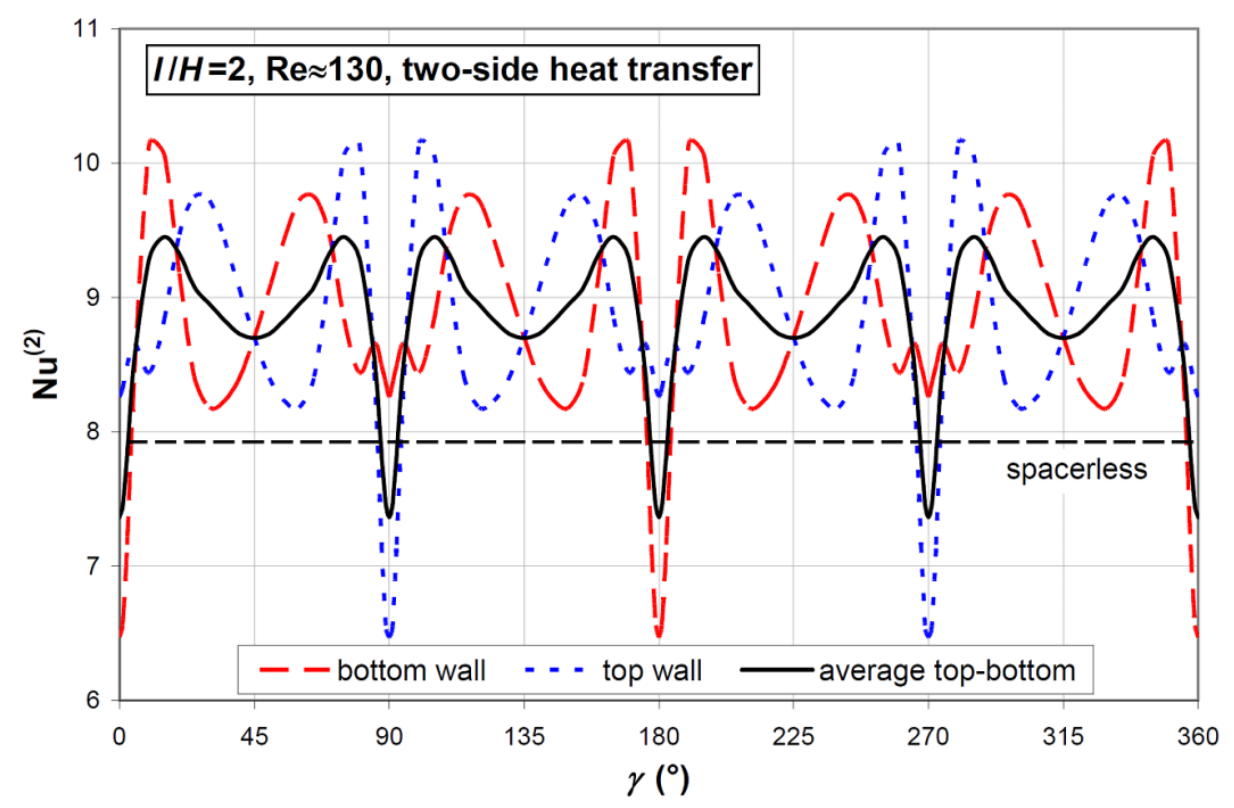

(b)

Figure 9 Influence of the flow attack angle on the average Nusselt number $\mathrm{Nu}^{(2)}$ on the top and bottom walls for an overlapped spacer filled channel with $l / H=2, \mathrm{Re} \approx 130$, two-side heat transfer. (a) Detail of $\mathrm{Nu}^{(2)}$ top, $\mathrm{Nu}^{(2)}$ bottom, and top-bottom average for $\gamma=0-\pi / 4$; (b) overall behavior for $\gamma=0-2 \pi$. 
For example, Figure 9 reports the average Nusselt number $\mathrm{Nu}^{(2)}$ as a function of $\gamma$ for the top and the bottom walls of an overlapped spacer-filled channel characterized by $l / H=2, \operatorname{Re} \approx 130$. The top-bottom average is also reported. Graph (a) shows in detail the interval $\gamma=0-\pi / 4$, whereas graph (b) (built from graph (a) using the above symmetry properties) spans the whole interval $\gamma=0-2 \pi$. It can be observed that both the individual top and bottom wall averages, and the overall average, exhibit a complex behavior and are not monotonic functions of $\gamma$ in the base interval $0-\pi / 4\left(0-45^{\circ}\right)$.

In particular, in the present case the overall average is minimum ( 7.4) for $\gamma=0$, due to the deep minimum exhibited by the average $\mathrm{Nu}$ on the bottom-wall, where the flow is orthogonal to the filaments, and is maximum ( $\sim 9.4)$ for $\gamma \approx 15^{\circ}$, mainly due to the high heat transfer rates attained, again on the bottom wall, for this flow orientation. The variance of the overall (top-bottom) average with $\gamma$ is necessarily lower than that exhibited by each wall average, but is still quite significant.

Figure 10 reports the friction coefficient $f$ (defined on the basis of the hydraulic diameter of the spacerless channel, $2 H$, and on the corresponding velocity $u_{v o i d}$ ) as a function of $\gamma$ for the same configuration in Figure 9, i.e. $l / H=2, \operatorname{Re} \approx 130$ (in this case thermal boundary conditions are, of course, immaterial). It can be observed that $f$ attains a maximum for $\gamma \approx 15^{\circ}$, in approximate correspondence with the heat transfer maximum. Obviously no simple correlation, let alone a power-law one, can capture the complex behaviour of either $\mathrm{Nu}$ or $f$ as functions of $\gamma$.

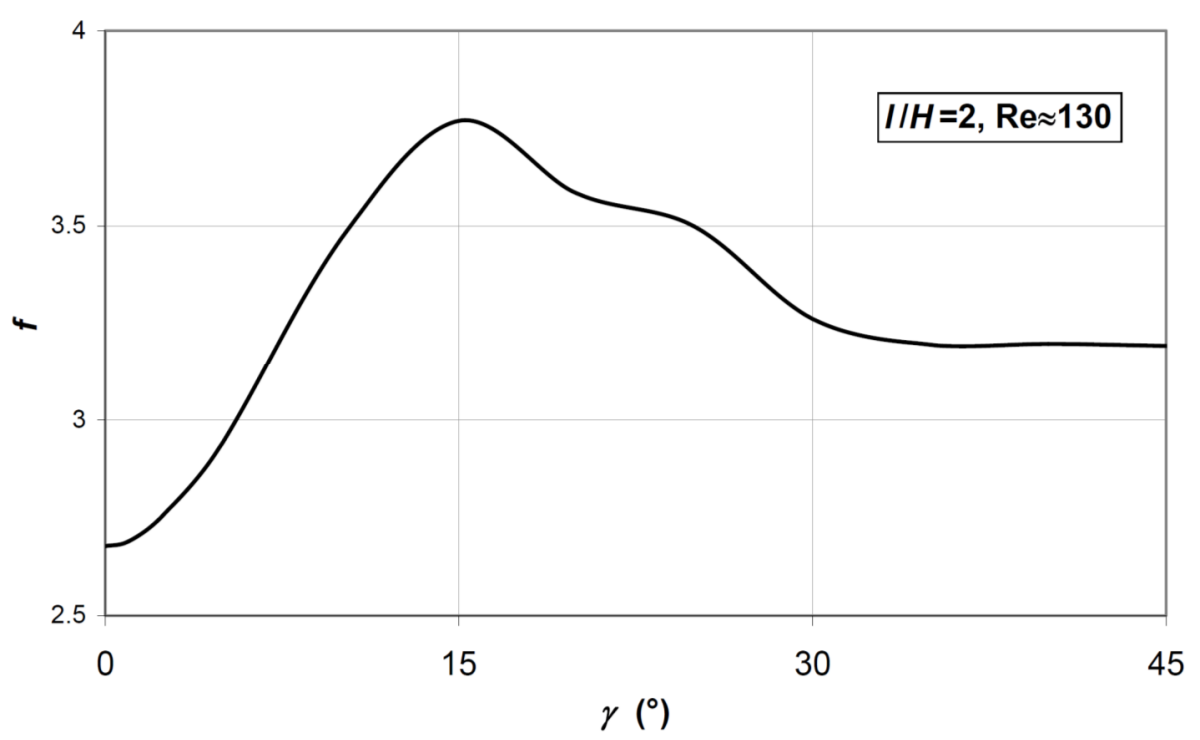

Figure 10 Influence of the flow attack angle on the Darcy friction coefficient $f$ for an overlapped spacer filled channel with $l / H=2, \operatorname{Re} \approx 130$, two-side heat transfer, $R \approx 1$. 


\section{INFLUENCE OF THE THERMAL CONDUCTIVITY OF THE SPACER}

In MD, spacer filaments are usually made of some polymeric material whose thermal conductivity $\left(\lambda_{s p a} \approx 0.12-0.20 \mathrm{Wm}^{-1} \mathrm{~K}^{-1}\right)$, albeit low, is not negligible with respect to the thermal conductivity of water $\left(\lambda_{f} \approx 0.6 \mathrm{Wm}^{-1} \mathrm{~K}^{-1}\right)$. Therefore, conduction through the spacer is expected to have some influence on heat transfer and thus on the Nusselt number. These effects are implicitly taken into account in experimental work (provided the same spacer material is adopted as in real applications), but in computational work the issue is generally neglected: spacers are simulated as non conducting regions or even as non-gridded regions external to the computational domain, and zero heat flux conditions are imposed at the fluid-spacer interfaces.

In the present study, some simulations were run with the spacer filaments included in the computational domain and gridded with finite volumes, and continuity of temperature and normal heat flux imposed at the fluid-spacer interface. The conductivity ratio $\lambda_{s p a} / \lambda_{f}$ was made to vary between 0.01 (equivalent to non conducting filaments, and chosen only as a consistency test) and 4 (realizable, for example, by using plastics charged with a suitable filler). Note that common practical applications correspond to $\lambda_{s p a} / \lambda_{f} \approx 0.25$.

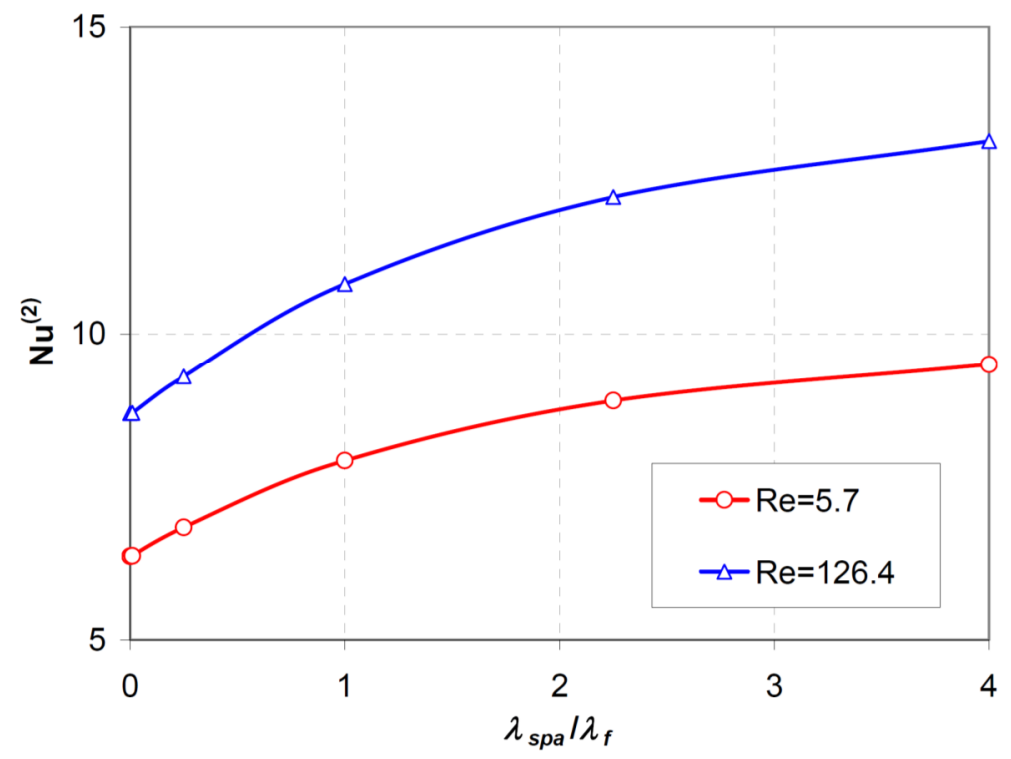

Figure 11 Influence of the thermal conductivity of the spacer: Nusselt number $\mathrm{Nu}^{(2)}$ as a function of the conductivity ratio $\lambda_{\text {spa }} / \lambda_{f}$ for an overlapped configuration with $l / H=2, \gamma=45^{\circ}$, two-side heat transfer. Note that $\mathrm{Nu}^{(2)}{ }_{\text {top }}=\mathrm{Nu}^{(2)}{ }_{\text {bottom }}$ for this flow attack angle.

Figure 11 shows the average Nusselt number $\mathrm{Nu}^{(2)}$ as a function of the ratio $\lambda_{\text {spal }} / \lambda_{f}$ for two values of the Reynolds number and for $l / H=2$, two-side heat transfer, $\gamma=45^{\circ}$ (note that $\mathrm{Nu}^{(2)}{ }_{\text {top }}=\mathrm{Nu}^{(2)}$ bottom for this flow attack angle). The Nusselt number increases considerably with the conductivity ratio: even 
for a realistic spacer conductivity $\left(\lambda_{\text {spal }} / \lambda_{f}=0.25\right), \mathrm{Nu}^{(2)}$ values are some $7 \%$ higher than in the nonconductive case, and the increment rises to $\sim 50 \%$ in the case of high-conductivity filaments $\left(\lambda_{\text {spa }} / \lambda_{\mathrm{f}}=4\right)$. Interestingly, the relative increment of $\mathrm{Nu}^{(2)}$ depends little on the Reynolds number.

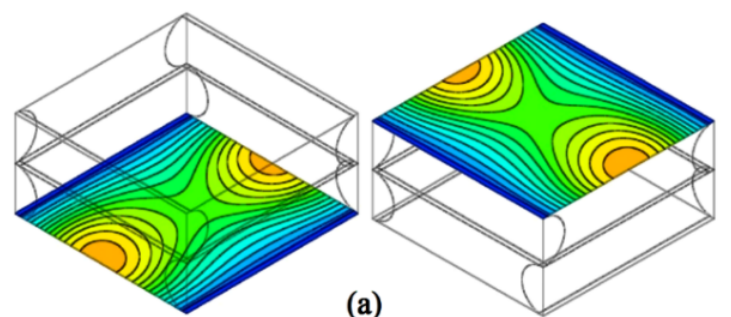

(a)

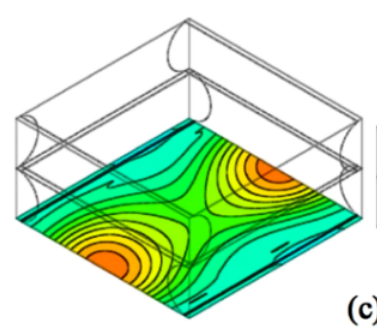

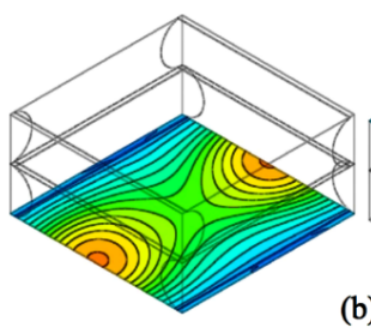

(b)
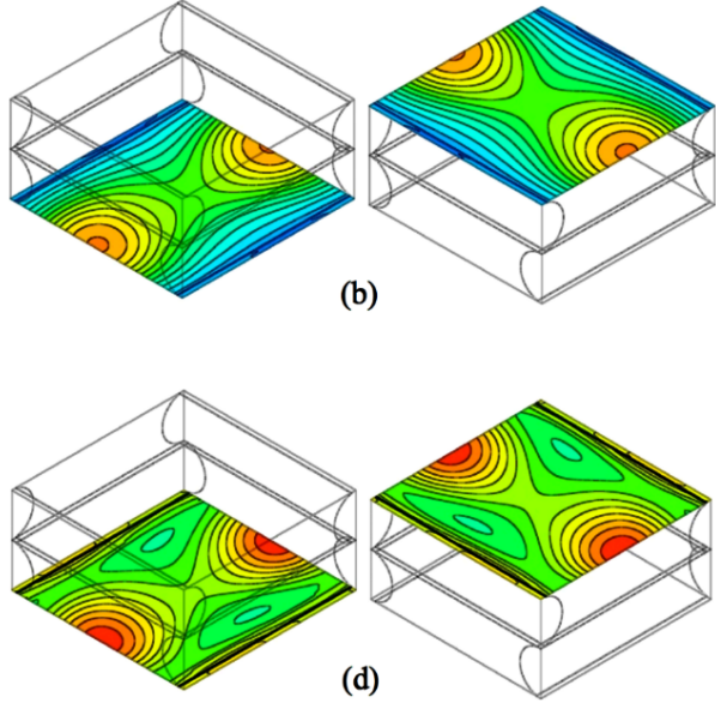

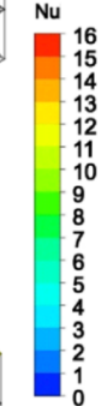

(d)

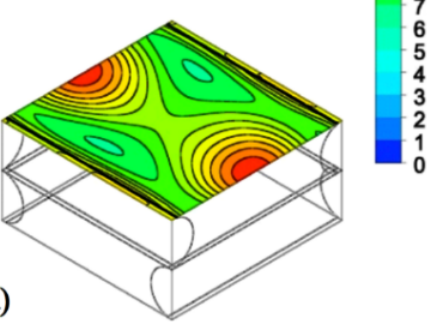

Figure 12 Top and bottom wall distributions of the local Nusselt number for an overlapped spacer configuratiuon with $l / H=2, \theta=45^{\circ}, \operatorname{Re}=5.7$, two-side heat transfer and different values of the thermal conductivity of the spacer filaments. (a) $\lambda_{s p a} / \lambda_{f}=0.01$; (b) $\lambda_{\text {spa }} / \lambda_{f}=0.25$; (c) $\lambda_{\text {spa }} / \lambda_{f}=1$; (d) $\lambda_{s p a} / \lambda_{f}=4$.

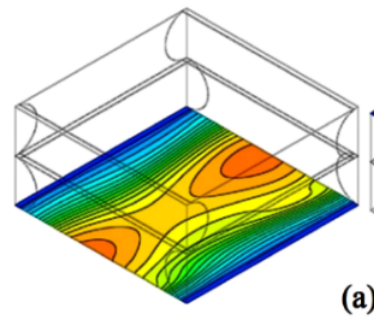

(a)
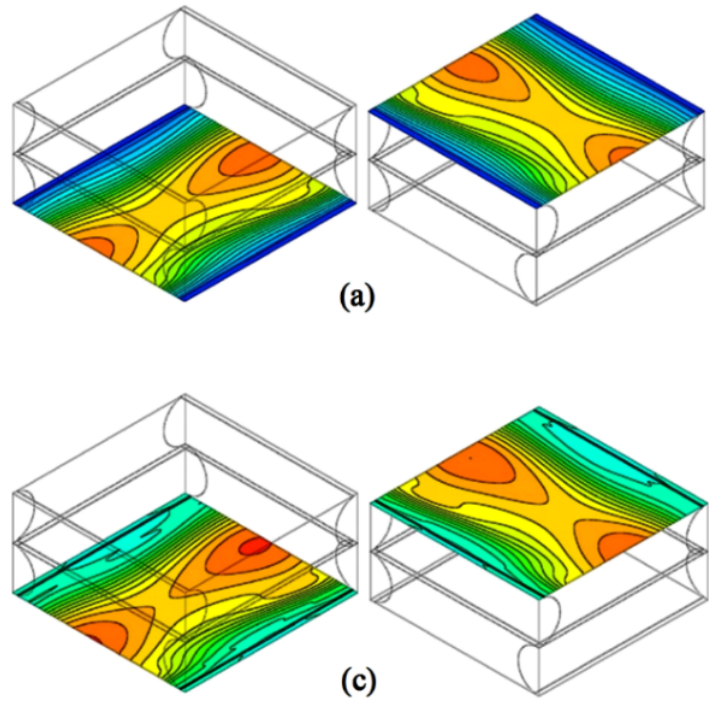
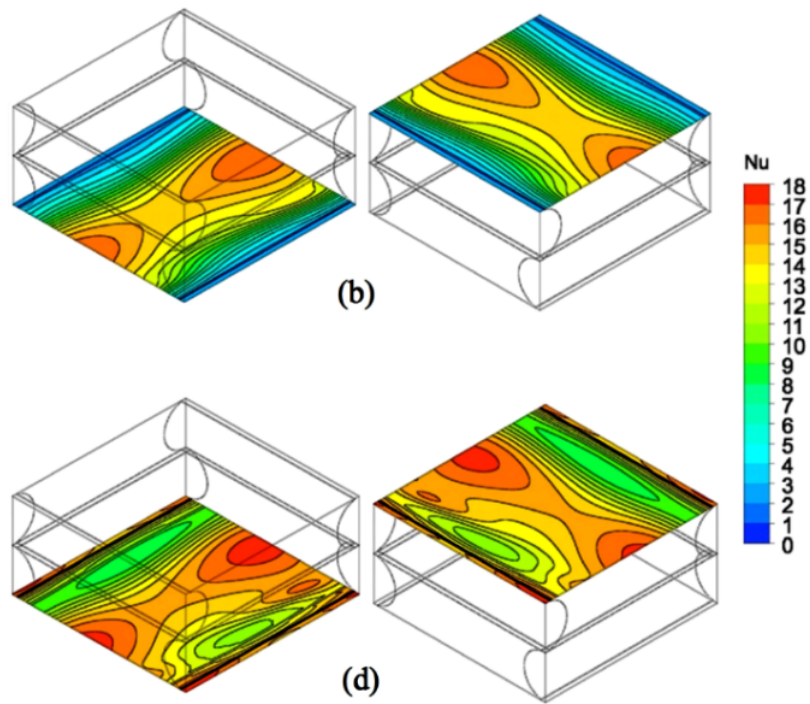

Figure 13 Top and bottom wall distributions of the local Nusselt number for an overlapped spacer configuratiuon with $l / H=2, \theta=45^{\circ}, \operatorname{Re}=126.4$, two-side heat transfer and different values of the thermal conductivity of the spacer filaments. (a) $\lambda_{\text {spa }} / \lambda_{\mathrm{f}}=0.01$; (b) $\lambda_{\text {spa }} / \lambda_{\mathrm{f}}=0.25$; (c) $\lambda_{\text {spa }} / \lambda_{\mathrm{f}}=1$; (d) $\lambda_{\text {spa }} / \lambda_{\mathrm{f}}=4$.

Figures 12 and 13 report maps of the local Nusselt number $\mathrm{Nu}$ on the top and bottom walls corresponding to four different values of the conductivity ratio $(0.01,0.25,1$ and 4$)$ for the same 
configuration $\left(l / H=2, \gamma=45^{\circ}\right.$, two-side heat transfer $)$ and $\mathrm{Re}=5.7$ and 126.4 , respectively. They show that the presence of the conductive filaments not only leads to an increase of the local and average $\mathrm{Nu}$ values but also produces a profound change in the distribution of $\mathrm{Nu}$; in particular, as expected, the low values of $\mathrm{Nu}$ in correspondence with the wall-spacer contact areas increase considerably with $\lambda_{\text {spal }} / \lambda_{f}$, while maxima are less affected.

At least two conclusions can be drawn from the above results:

I) In comparative studies between experimental results and CFD predictions, these latter should include the simulation of conduction in the spacer filaments since it is typically responsible for 5$10 \%$ of the overall heat flow.

II) Leaving cost considerations apart, the use of conductive spacers might be beneficial in MD. Of course, not all of the thermal power transferred from the hot bulk fluid to the conducting spacer can be regarded as "useful". In fact, only the fraction transferred back to the near-wall, colder fluid results in an increased vapor mass flux through the membrane, whereas the fraction that is directly transferred from the spacer to the membrane by conduction through the contact spots can actually be detrimental, as it increments the conductive losses that reduce the feed temperature without producing any permeate. Therefore, the net effect of the spacer conductivity depends on the exact geometry of the spacer and of the spacer-membrane contact areas, and some considerable CFD and experimental work is probably required before definite conclusions on this issue can be drawn.

\section{CONCLUSIONS}

The main objective of the present paper was to clarify a number of issues arising in the study of fluid flow and heat/mass transfer in Membrane Distillation (MD) and other membrane processes involving flat, spacer-filled, channels (either plane or spirally wound). Other designs, including dead-end arrangements and tubular or hollow-fiber membranes, raise different problems which were not discussed here. The conclusions reached were based on many years' experience with MD, both experimental and computational.

The results of the study can be summarized as follows:

- In regard to the choice of the scales for data reduction and for the definition of dimensionless numbers, one should keep in mind that several options have been adopted in the literature, and that they should be carefully considered whenever comparisons of data from different sources are to be performed.

- In regard to the definition of local heat or mass transfer coefficients, the definition based on the local heat / mass flux and on the difference between the local temperature or concentration at the wall and the corresponding bulk values, i.e. $h=q{ }^{\prime \prime} /\left(T_{b}-T_{w}\right)$ or $k=j_{w} /\left(C_{b}-C_{w}\right)$, remains the most 
commonly adopted, but we have shown that these quantities may become singular or negative at some points of the wall in complex geometries. As a consequence, the definition of the average coefficients as surface averages $h^{(1)}=\langle h\rangle, k^{(1)}=\langle k\rangle$ (definitions 1) may lead to singularities. Alternative definitions such as $h^{(2)}=\left\langle q^{\prime \prime}{ }_{w}\right\rangle\left(\left(T_{b}-\left\langle T_{w}\right\rangle\right), k^{(2)}=\left\langle j_{w}\right\rangle /\left(C_{b}-\left\langle C_{w}\right\rangle\right)\right.$ (definitions 2) are more robust in that they do not suffer from singularities.

- In regard to the influence of thermal conduction in the spacer, numerical simulations performed by including this phenomenon in the computational model have shown that even lowconductivity polymeric spacers $\left(\lambda \approx 0.15 \mathrm{Wm}^{-1} \mathrm{~K}^{-1}\right)$ may be responsible for up to $10 \%$ of the total heat transferred from the fluid to the membrane. Therefore, the comparison of experimental results with CFD simulations neglecting spacer conduction is questionable. Our results also show that significant increments of heat flow would occur by using relatively high-conductivity spacers (e.g. $\lambda=2-10 \mathrm{Wm}^{-1} \mathrm{~K}^{-1}$ ), making this possibility at least worth investigating in greater detail.

- Finally, in regard to the complex dependence of heat / mass transfer and pressure drop upon the physical and geometrical parameters that characterize the spacer-filled channels, an analysis was presented for overlapped spacers with orthogonal filaments. The Nusselt and Sherwood number $\mathrm{Nu}^{(2)}$ and $\mathrm{Sh}^{(2)}$ on either side varied in a complex way with the Reynolds number, exhibiting in some cases a plateau in an interval of $\operatorname{Re}$ (for example, this was between $\operatorname{Re} \approx 100$ and $\operatorname{Re} \approx 300$ for $l / H=2.5$ and $\gamma=20^{\circ}$ ); the friction coefficient $f$ decreased monotonically with Re but did not follow a simple power-law dependence. When the spacer aspect ratio $l / H$ was made to vary, for sufficiently high Reynolds number $\mathrm{Nu}^{(2)}$ and $\mathrm{Sh}^{(2)}$ exhibited a maximum for $l / H \approx 3.5$, while $f$ decreased monotonically. Finally, $\mathrm{Nu}^{(2)}$ and $\mathrm{Sh}^{(2)}$ varied in a complex way with the flow attack angle, exhibiting in some cases multiple maxima and minima even in the basic interval $\gamma=0-\pi / 4$; similar variations were also exhibited by the friction coefficient $f$. Notably, the three parameters Re, $l / H$ and $\gamma$ were found to interact heavily, making a separate-effect analysis impossible and power-law friction of heat / mass transfer correlations of little use. For any optimization study seeking the maximum of some objective function as the parameters are made to vary, interpolation from an adequately large and finely stepped database of Nusselt / Sherwood numbers and friction coefficients is probably to be preferred to the use of correlations.

A number of issues have intentionally been left out of the present study, and may be the subject of further work. Among them, the influence of boundary conditions for temperature and concentration; the influence of entry effects in complex, e.g. spacer-filled, geometries; and the subject of unsteadiness and turbulence modeling in MD and related problems. 


\section{Acknowledgements}

This work was funded within the EU FP7 programme by projects MEDIRAS (contract number 218938) and REAPower (contract number 256736) and within the Horizon 20-20 programme by project RED-HtP (contract number 640667). 


\begin{tabular}{|c|c|c|}
\hline Symbol & Quantity & Unit \\
\hline$A$ & Cross sectional area & $\mathrm{m}^{2}$ \\
\hline$C$ & Concentration & $\mathrm{mol} \mathrm{m}^{-3}$ \\
\hline$D$ & Kinematic diffusivity & $\mathrm{m}^{2} \mathrm{~s}^{-1}$ \\
\hline$d_{f}$ & Spacer filament diameter $(\approx H / 2)$ & $\mathrm{m}$ \\
\hline$d_{h}$ & Spacer hydraulic diameter, $4 V_{f} / S_{\text {wet }}$ & $\mathrm{m}$ \\
\hline$f$ & Darcy friction coefficient & - \\
\hline$H$ & Channel height & $\mathrm{m}$ \\
\hline$h$ & Heat transfer coefficient, $q^{\prime \prime}{ }_{w} /\left(T_{b}-T_{w}\right)$ & $\mathrm{W} \mathrm{m}^{-2} \mathrm{~K}^{-1}$ \\
\hline$j$ & Mass flux & $\mathrm{mol} \mathrm{m}^{-2} \mathrm{~s}^{-1}$ \\
\hline$k$ & Mass transfer coefficient, $j_{w} /\left(C_{b^{-}} C_{w}\right)$ & $\mathrm{m} \mathrm{s}^{-1}$ \\
\hline$l$ & Spacer filament pitch & $\mathrm{m}$ \\
\hline $\mathrm{Nu}$ & Nusselt number, $h \cdot 2 H / \lambda$ & - \\
\hline $\operatorname{Pr}$ & Prandtl number, $c_{p} \mu / \lambda$ & - \\
\hline$Q$ & Volume flow rate & $\mathrm{m}^{3} \mathrm{~s}^{-1}$ \\
\hline$Q_{1}$ & Volume flow rate per unit width & $\mathrm{m}^{2} \mathrm{~s}^{-1}$ \\
\hline$q "$ & Heat flux & $\mathrm{W} \mathrm{m}^{-2}$ \\
\hline$r_{e x t}$ & Thermal resistance at the wall & $\mathrm{m}^{2} \mathrm{~K} \mathrm{~W}^{-1}$ \\
\hline $\operatorname{Re}$ & Bulk Reynolds number, $u_{\text {void }} 2 H / v$ & - \\
\hline$S$ & Surface area & $\mathrm{m}^{2}$ \\
\hline$s$ & Co-ordinate along the main flow direction & $\mathrm{m}$ \\
\hline $\mathrm{Sc}$ & Schmidt number, $v / D$ & - \\
\hline $\mathrm{Sh}$ & Sherwood number, $k \cdot 2 H / D$ & - \\
\hline$T$ & Temperature & $\mathrm{K}$ \\
\hline$U$ & Overall heat transfer coefficient, $q{ }^{\prime}{ }_{w} /\left(T_{b}-T_{e x t}\right)$ & $\mathrm{W} \mathrm{m}{ }^{-2} \mathrm{~K}^{-1}$ \\
\hline$u_{\text {void }}$ & Mean velocity in the void channel, $Q /(H W)$ & $\mathrm{m} \mathrm{s}^{-1}$ \\
\hline$u_{m}$ & Mean velocity in the spacer-filled channel & $\mathrm{m} \mathrm{s}^{-1}$ \\
\hline$V$ & Volume & $\mathrm{m}^{3}$ \\
\hline$W$ & Channel width (spanwise extent) & $\mathrm{m}$ \\
\hline
\end{tabular}


Greek symbols

$\begin{array}{ll}\alpha & \text { Included angle } \\ \gamma & \text { Flow attack angle } \\ \delta & \text { Channel half-thickness } \\ \varepsilon & \text { Porosity, or void ratio, } V_{f} / V_{\text {tot }} \\ \lambda & \text { Thermal conductivity } \\ \mu & \text { Viscosity } \\ \nu & \text { Kinematic viscosity } \\ \rho & \text { Density }\end{array}$

deg or radians deg or radians

m

$\mathrm{W} \mathrm{m}{ }^{-1} \mathrm{~K}^{-1}$

$\mathrm{kg} \mathrm{m}^{-1} \mathrm{~s}^{-1}$

$\mathrm{m}^{2} \mathrm{~s}^{-1}$

$\mathrm{kg} \mathrm{m}^{-3}$

\section{Subscripts}

$b$

f

$S$

spa

tot

w

wet

Averages

$\langle\Phi\rangle$
Bulk

Fluid

Main flow direction

Spacer

Total

Wall

Wetted

Surface average of quantity $\Phi$ 


\section{REFERENCES}

[1] P. Onsekizoglu, Membrane Distillation: principle, advances, limitations and future prospects in food industry, in Distillation-Advances from Modeling to Applications, Zereshki S. (Ed.), InTech (2012).

[2] A. Cipollina, A. Di Miceli, J. Koschikowski, G. Micale, L. Rizzuti, CFD simulation of a Membrane Distillation module channel, Desalination and Water Treatment 6 (2009) 177-183.

[3] A. Cipollina, G. Micale, L. Rizzuti, Membrane Distillation heat transfer enhancement by CFD analysis of internal module geometry, Desalination and Water Treatment 25 (2011) 195-209.

[4] A. Tamburini, M. Renda, A. Cipollina, G. Micale, M. Ciofalo, Investigation of heat transfer in spacer-filled channels by experiments and direct numerical simulations, International Journal of Heat and Mass Transfer 93 (2016) 1190-1205.

[5] A. Tamburini, P. Pitò, A. Cipollina, G. Micale, M. Ciofalo, A Thermochromic Liquid Crystals image analysis technique to investigate temperature polarization in spacer-filled channels for Membrane Distillation, Journal of Membrane Science 447 (2013) 260-273.

[6] A. Tamburini, A. Cipollina, S. Al-Sharif, M. Albeyrutty, L. Gurreri, G. Micale, M. Ciofalo, Assessment of temperature polarization in Membrane Distillation channels by liquid crystal thermography, Desalination and Water Treatment 55 (2015) 2747-2765.

[7] A. Ali, F. Macedonio, E. Drioli, S. Aljlil, O. A. Alharbi, Experimental and theoretical evaluation of temperature polarization phenomenon in Direct Contact Membrane Distillation, Chemical Engineering Research and Design 91 (2013) 1966-1977.

[8] Z. Cao, D. E. Wiley, A. G. Fane, CFD simulations of net-type turbulence promoters in a narrow channel, Journal of Membrane Science 185 (2001) 157-176.

[9] M. Qureshi, M. Shakaib, CFD study for temperature and concentration profiles in membrane channels, Proceedings of International Conference on Energy and Sustainability, NED University of Engineering \& Technology, Karachi, Pakistan (2013).

[10] G. Schock, A. Miquel, Mass transfer and pressure loss in spiral wound modules, Desalination 64 (1987) 339-352.

[11] M. Shakaib, S. M. F. Hasani, I. Ahmed, R. M. Yunus, A CFD study on the effect of spacer orientation on temperature polarization in Membrane Distillation modules, Desalination 284 (2012) 332-340. 
[12] S. K. Karode, A. Kumar, Flow visualization through spacer filled channels by computational fluid dynamics I. Pressure drop and shear rate calculations for flat sheet geometry, Journal of Membrane Science 193 (2001) 69-84.

[13] A. R. Da Costa, A. G. Fane, D. E. Wiley, Spacer characterization and pressure drop modelling in spacer-filled channels for Ultra-Filtration, Journal of Membrane Science 87 (1994) 79-98.

[14] F. Li, W. Meindersma, A. B. de Haan, T. Reith, Optimization of commercial net spacers in spiral wound membrane modules, Journal of Membrane Science 208 (2002) 289-302.

[15] C. P. Koutsou, S. G. Yiantsios, A. J. Karabelas, Direct numerical simulation of flow in spacerfilled channels: effect of spacer geometrical characteristics, Journal of Membrane Science 291 (2007) 53-69.

[16] C. P. Koutsou, S. G. Yiantsios, A. J. Karabelas, A numerical and experimental study of mass transfer in spacer-filled channels: effects of spacer geometrical characteristics and Schmidt number, Journal of Membrane Science 326 (2009) 234-251.

[17] C. P. Koutsou, A. J. Karabelas, A novel retentate spacer geometry for improved spiral wound membrane (SWM) module performance, Journal of Membrane Science 488 (2015) 129-142.

[18] S. Al-Sharif, M. Albeirutty, A. Cipollina, G. Micale, Modelling flow and heat transfer in spacerfilled Membrane Distillation channels using open source CFD code, Desalination 311 (2013) 103112.

[19] S. M. Mojab, A. Pollard, J. G. Pharoah, S. B. Beale, E. S. Hanff, Unsteady laminar to turbulent flow in a spacer-filled channel, Flow, Turbulence \& Combustion 92 (2014) 563-577.

[20] A. Saeed, R. Vuthaluru, H. B. Vuthaluru, Investigations into the effects of mass transport and flow dynamics of spacer filled membrane modules using CFD, Chemical Engineering Research and Design 93 (2015) 79-99.

[21] S. S. Bucs, R. V. Linares, J. O. Marston, A. I. Radu, J. S. Vrouwenvelder, C. Picioreanu, Experimental and numerical characterization of the water flow in spacer-filled channels of spiralwound membranes, Water Research 87 (2015) 299-310.

[22] L. Gurreri, A. Tamburini, A. Cipollina, G. Micale, M. Ciofalo, Flow and mass transfer in spacerfilled channels for Reverse Electrodialysis: a CFD parametrical study, Journal of Membrane Science 497 (2016) 300-317.

[23] J. Schwinge, P. R. Neal, D. E. Wiley, D. F. Fletcher, A. G. Fane, Spiral wound modules and spacers - Review and analysis, Journal of Membrane Science 242 (2004) 129-153. 
[24] R. Ghidossi, D. Veyret, P. Moulin, Computational fluid dynamics applied to membranes: State of the art and opportunities, Chemical Engineering and Processing 45 (2006) 437-454.

[25] I. Hitsov, T. Maere, K. DeSitter, C. Dotremont, I. Nopens, Modelling approaches in Membrane Distillation: a critical review, Separation and Purification Technology 142 (2015) 48-64.

[26] G. A. Fimbres-Weihs, D. E. Wiley, Review of 3D CFD modeling of flow and mass transfer in narrow spacer-filled channels in membrane modules, Chemical Engineering and Processing 49 (2010) 759-781.

[27] A. J. Karabelas, M. Kostoglou, C. P. Koutsou, Modeling of spiral wound membrane desalination modules and plants: review and research priorities, Desalination 356 (2015) 165-186.

[28] J. Phattaranawik, R. Jiraratananon, A. G. Fane, Effects of net-type spacers on heat and mass transfer in direct contact membrane distillation and comparison with Ultrafiltration studies, Journal of Membrane Science 217 (2003) 193-206. 